\title{
Slower EEG alpha generation, synchronization and "flow" - possible biomarkers of cognitive impairment and neuropathology of minor stroke
}

\author{
Jelena Petrovic ${ }^{\text {Corresp., }}{ }^{1}$, Vuk Milosevic ${ }^{2}$, Miroslava Zivkovic ${ }^{2}$, Dragan Stojanov ${ }^{3}$, Olga Milojkovic $^{4}$, \\ Aleksandar Kalauzi ${ }^{5}$, Jasna Saponjic ${ }^{1}$ \\ ${ }^{1}$ Department of Neurobiology, Institute for Biological Research - Sinisa Stankovic, University of Belgrade, Belgrade, Serbia \\ 2 Clinic of Neurology, Clinical Center Nis, Serbia, Nis, Serbia \\ 3 Institute of Radiology, Clinical Center Nis, Nis, Serbia \\ ${ }^{4}$ Clinic for Mental Health Protection, Clinical Center Nis, Nis, Serbia \\ 5 Department for Life Sciences, Institute for Multidisciplinary Research, University of Belgrade, Belgrade, Serbia \\ Corresponding Author: Jelena Petrovic \\ Email address: jelena.petrovic@ibiss.bg.ac.rs
}

Background: We investigated EEG rhythms, particularly alpha activity, and their relationship to post-stroke neuropathology and cognitive functions in the subacute and chronic stages of minor strokes. Methods: We included 10 patients with right middle cerebral artery (MCA) ischemic strokes and 11 healthy controls. All the assessments of stroke patients were done both in the subacute and chronic stages. Neurological impairment was measured using the National Institute of Health Stroke Scale (NIHSS), whereas cognitive functions were assessed using the Montreal Cognitive Assessment (MoCA) and MoCA memory index (MoCA-MIS). The EEG was recorded using a 19 channel EEG system with standard EEG electrode placement. In particular, we analyzed the EEGs derived from the four lateral frontal $(\mathrm{F} 3, \mathrm{~F} 7, \mathrm{~F} 4, \mathrm{~F} 8)$, and corresponding lateral posterior (P3,P4,T5,T6) electrodes. Quantitative EEG analysis included: the group FFT spectra, the weighted average of alpha frequency ( $A A V G$ ), the group probability density distributions of all conventional EEG frequency band relative amplitudes (EEG microstructure), the interand intra-hemispheric coherences, and the topographic distribution of alpha carrier frequency phase potentials (PPs). Statistical analysis was done using a Kruskal-Wallis ANOVA with a post-hoc Mann-Whitney U two-tailed test, and Spearman's correlation. Results: We demonstrated transient cognitive impairment alongside a slower alpha frequency ( $\alpha \mathrm{AVG}$ ) in the subacute right MCA stroke patients vs. the controls. This slower alpha frequency showed no amplitude change, but was highly synchronized intrahemispherically, overlying the ipsi-lesional hemisphere, and inter-hemispherically, overlying the frontal cortex. In addition, the disturbances in EEG alpha activity in subacute stroke patients were expressed as a decrease in alpha PPs over the frontal cortex and an 
altered "alpha flow", indicating the sustained augmentation of inter-hemispheric interactions. Although the stroke induced slower alpha was a transient phenomenon, the increased alpha intra-hemispheric synchronization, overlying the ipsi-lesional hemisphere, the increased alpha F3-F4 inter-hemispheric synchronization, the delayed alpha waves, and the newly established inter-hemispheric "alpha flow" within the frontal cortex, remained as a permanent consequence of the minor stroke. This newly established frontal inter-hemispheric "alpha flow" represented a permanent consequence of the "hidden" stroke neuropathology, despite the fact that cognitive impairment has been returned to the control values. All the detected permanent changes at the EEG level with no cognitive impairment after a minor stroke could be a way for the brain to compensate for the lesion and restore the lost function. Discussion: Our study indicates slower EEG alpha generation, synchronization and "flow" as potential biomarkers of cognitive impairment onset and/or compensatory post-stroke re-organizational processes. 
1

2

3

4

5

7

8

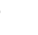

\section{*Corresponding author:} 6 Aleksandar Kalauzi ${ }^{5}$, Jasna Saponjic ${ }^{1}$

\section{Slower EEG alpha generation, synchronization and "flow" - possible biomarkers of cognitive impairment and neuropathology of minor stroke}

Jelena Petrovic $^{1 *}$, Vuk Milosevic ${ }^{2}$, Miroslava Zivkovic ${ }^{2}$, Dragan Stojanov³ ${ }^{3}$ Olga Milojkovic ${ }^{4}$,

${ }^{1}$ University of Belgrade, Department of Neurobiology, Institute for Biological Research - Sinisa Stankovic, Belgrade, Serbia; ${ }^{2}$ Clinic of Neurology, Clinical Center Nis, Serbia; ${ }^{3}$ Institute of Radiology, Clinical Center Nis, Serbia; ${ }^{4}$ Clinic for Mental Health Protection, Clinical Center Nis, Serbia; 5 University of Belgrade, Department for Life Sciences, Institute for Multidisciplinary Research, Belgrade, Serbia.

Jelena Petrovic, Ph.D.

Department of Neurobiology, Institute for Biological Research - Sinisa Stankovic, Despot Stefan Blvd., 142 11060 Belgrade, Serbia. (Phone) + 381112078426 (Fax) + 381112761433

e-mail: jelena.petrovic@ibiss.bg.ac.rs 


\section{Abstract}

Background: We investigated EEG rhythms, particularly alpha activity, and their relationship to post-stroke neuropathology and cognitive functions in the subacute and chronic stages of minor strokes.

Methods: We included 10 patients with right middle cerebral artery (MCA) ischemic strokes and 11 healthy controls. All the assessments of stroke patients were done both in the subacute and chronic stages. Neurological impairment was measured using the National Institute of Health Stroke Scale (NIHSS), whereas cognitive functions were assessed using the Montreal Cognitive Assessment (MoCA) and MoCA memory index (MoCA-MIS). The EEG was recorded using a 19 channel EEG system with standard EEG electrode placement. In particular, we analyzed the EEGs derived from the four lateral frontal (F3, F7, F4, F8), and corresponding lateral posterior (P3, P4, T5, T6) electrodes. Quantitative EEG analysis included: the group FFT spectra, the weighted average of alpha frequency (aAVG), the group probability density distributions of all conventional EEG frequency band relative amplitudes (EEG microstructure), the inter- and intrahemispheric coherences, and the topographic distribution of alpha carrier frequency phase potentials (PPs). Statistical analysis was done using a Kruskal-Wallis ANOVA with a post-hoc Mann-Whitney U two-tailed test, and Spearman's correlation.

Results: We demonstrated transient cognitive impairment alongside a slower alpha frequency $(\alpha \mathrm{AVG})$ in the subacute right MCA stroke patients vs. the controls. This slower alpha frequency showed no amplitude change, but was highly synchronized intra-hemispherically, overlying the ipsi-lesional hemisphere, and inter-hemispherically, overlying the frontal cortex. In addition, the disturbances in EEG alpha activity in subacute stroke patients were expressed as a decrease in alpha PPs over the frontal cortex and an altered "alpha flow", indicating the sustained 
49 augmentation of inter-hemispheric interactions. Although the stroke induced slower alpha was a

50 transient phenomenon, the increased alpha intra-hemispheric synchronization, overlying the ipsi-

51 lesional hemisphere, the increased alpha F3-F4 inter-hemispheric synchronization, the delayed

52 alpha waves, and the newly established inter-hemispheric "alpha flow" within the frontal cortex,

53 remained as a permanent consequence of the minor stroke. This newly established frontal inter-

54 hemispheric "alpha flow" represented a permanent consequence of the "hidden" stroke

55 neuropathology, despite the fact that cognitive impairment has been returned to the control

56 values. All the detected permanent changes at the EEG level with no cognitive impairment after

57 a minor stroke could be a way for the brain to compensate for the lesion and restore the lost

58 function.

59 Discussion: Our study indicates slower EEG alpha generation, synchronization and "flow" as

60 potential biomarkers of cognitive impairment onset and/or compensatory post-stroke re61 organizational processes. 


\section{Introduction}

Stroke is a common neurological disease with variable outcomes regarding the level of disabilities and mortality caused (Strum et al., 2002). In most cases (80-90\%) a stroke occurs as a result of reduced blood flow, due to an obstructed blood vessels (Grefkes and Ward, 2014), causing deficits in a number of neurological domains, including cognitive impairment (Cramer, 2008). The initial and sudden cognitive decline after a stroke is characterized by partial recovery within a few months (Thiel et al., 2014). However, cognitive assessment at least 3 months after a stroke shows more stable, long lasting cognitive impairment. Although varying between countries, races and diagnostic criteria, the prevalence of post-stroke cognitive impairment in stroke survivors is high. Between 7 and $41 \%$ of ischemic stroke patients have dementia 3 to 12 months after their stroke and more than $80 \%$ show cognitive impairment in one or multiple domains which does not fulfil the criteria for dementia (Jokinen et al., 2015, Mijajlovic et al., 2017, Pendlebury et al., 2009, Sun et al., 2014). Cognitive impairment significantly influences the independent functioning and quality of life of stroke patients. However, given the severity of the physical disabilities involved, post-stroke cognitive impairment is often neglected (Mijajlovic et al., 2017).

The post-stroke decline in general cognitive status occurs as a consequence of diverse cortical and subcortical strokes, with resulting neurocognitive deficits, reflecting the extent and location of the lesions (Sheorajpanday et al., 2014). The degree of deviation from normal cognition in stroke patients is highly variable in terms of both type and severity, since different domains, including executive function, attention, memory, visuospatial abilities, language and orientation, may be affected to varying degrees (Kalaria et al., 2016). All of these impairments, particularly subtle cognitive dysfunction, might easily go unnoticed without appropriate 
86 screening. The most commonly used method for measuring cognitive impairment is a

87 neuropsychological assessment which employs brief screening tests, such as the Mini Mental

88 State Examination (MMSE) (Folstein et al., 1975), and the Montreal Cognitive Assessment

89 (MoCA) (Nasreddine et al., 2005). Although widely accepted for cognitive assessment, none of

90 these tests are specifically designed for post-stroke cognitive screening. Therefore, despite their

91 ability to identify cognitive dysfunction, these tests have limitations regarding their sensitivity in

92 detecting all cases of cognitive impairment, their selectivity in ruling out those without cognitive

93 impairment, and finally, the types of stroke patients that can be assessed (Schleiger et al., 2014).

EEG is a low-cost, non-invasive imaging technique that has been used in clinical practice for decades. Having excellent temporal resolution, EEG provides a direct measurement of the cerebral functional status, with subtle EEG changes reflecting underlying pathophysiological processes (Borich et al., 2015). EEG abnormalities are typical manifestations of an ischemic stroke. Following cerebral blood flow reduction, the metabolic and electrical activities of cortical neurons are altered (George and Steinberg, 2015; Hossmann, 1994). These alterations can be observed as specific EEG patterns over the ischemic area, coming from the attenuation of faster (alpha and beta) and the augmentation of slower (delta and theta) frequency bands (Jordan, 2004; Nagata et al., 1989). Apart from being very sensitive in the detection of cerebral ischemia, EEG can also be used as a diagnostic and prognostic tool, or to track therapy during stroke recovery (Burghaus et al., 2013; Finnigan and van Putten, 2013; Finnigan et al., 2016; Foreman and Claassen, 2012). Namely, according to recent studies, the quantitative measures of specific EEG features (qEEG) are even more sensitive than raw EEG, and well correlated with stroke severity and radiographic findings (Finnigan et al., 2004; Sheorajpanday et al., 2011), response to 
108 treatment such as thrombolytic therapy (Finnigan et al., 2006), and to functional outcomes 109 (Burghaus et al., 2013; Finnigan et al., 2004).

In addition, many studies demonstrated that some qEEG indices are also sensitive to

111

112

113

114

115

116

117

118

119

120

121

122

123

124

125

126

127

128

129

130

cognitive function (Klimesh, 2012) and to mild cognitive impairment or dementia (Dubovik et al., 2013; Schleiger et al., 2014, 2017; Song et al., 2015). In line with the general view regarding cognitive and memory performance (Klimesch, 1999), these studies indicated that the alpha, as well as theta frequency, could be of interest for the screening of post-stroke cognitive impairment (Dubovik et al., 2013; Schleiger et al., 2014, 2017; Song et al., 2015). In contrast to relative theta power, whether global, as demonstrated by Song et al. (2015), or posterior, computed from three electrodes, as shown in Schleiger et al. (2017), the qEEG assessments revealed global alpha power (Schleiger et al., 2014), EEG alpha band synchrony (Dubovik et al., 2013), as well as a lower peak alpha frequency (Schleiger et al., 2017) to be informative as regards post-stroke cognitive impairment.

Since cortical activity varies during each stage of a stroke, the assessment of a stroke by EEG measurements has proven to be an essential monitoring standard for identifying spatiotemporal markers of cortical activity related to injury, the post-stroke re-organizational processes, and recovery (Iyer, 2017). The initial slowing of brain EEG activity in acute strokes could be characterized by analyzing the power of slower relative to faster EEG activities, such as the delta/alpha ratio (Finnigan et al., 2016; Schleiger et al., 2014). As the stroke progresses to the subacute and chronic stages, functional connectivity measures, such as phase synchrony within specific frequency band like alpha (Dubovik et al., 2012; Westlake et al., 2012), become more important for studying re-organizational processes, brain plasticity, and functional recovery (Iyer, 2017). 
All the abovementioned findings suggest that EEG measurements can be employed to

132

133

134

135

136

137

138

139

140

141

142

143

144

145

146

147

148

149

150

151

152

assess the general status of the brain and to complement neuropsychological testing when studying cognitive impairment following a stroke, potentially resulting in specific qEEG assesments that could be used as biomarkers of cognitive impairment and/or compensatory poststroke re-organizational processes. Therefore, the primary aim of this study is to investigate the alterations in EEG rhythms, and particularly in alpha activity, as a reflection of the underlying post-stroke neuropathology. Our secondary aim is to relate these EEG alterations to post-stroke re-organizational processes and cognitive functions in the subacute and chronic stages of a minor ischemic stroke vs. the controls.

\section{Materials and Methods}

\subsection{Subjects}

This research was conducted at the Clinic of Neurology of the Clinical Centre in Nis, Serbia. We included 21 subjects: 10 ischemic stroke patients and 11 healthy controls. One of the stroke patients died during the follow-up period. The clinical diagnosis of stroke and its classification was determined by a detailed patient history of symptoms, a neurological examination, and computed tomography (CT) and/or magnetic resonance imaging (MRI). Patients were assessed $9.70 \pm 2.50$ days following the onset of their stroke (the subacute stage for the first assessment), as well as $13.67 \pm 5.85$ months after their stroke (the chronic stage for the follow-up assessment). Neurological impairment was measured using the National Institute of Health Stroke Scale (NIHSS) on admission and at the time of both the first assessment and the follow-up assessment. Cognitive function was assessed by the Montreal Cognitive Assessment 
153 (MoCA) (Nasreddine et al., 2005) and the MoCA memory index score (MoCA-MIS) (Julayanont

154 et al., 2014) both in the subacute stage and during the follow up. The Edinburgh handedness 155 inventory-short form (EHI-SF) with four claims and a five-point response scale was used for 156 assessing hand dominance (Veale, 2014).

The inclusion criteria for the minor stroke patients were the following: the first ever

158

159

160

161

162

163

164

165

166

167

168

169

170

171

172

173

174

stroke, a confirmed ischemic stroke in the distribution of right middle cerebral artery (MCA), an

NIHSS of 4 or less, the absence of aphasia, right hand dominance (EHI-SF score $>60)$ and the absence of other neurological or psychiatric diseases. The control subjects were volunteers with no history of neurological or psychiatric disorders who were matched by age and years of formal education with the stroke group. We used the Informant Questionnaire on Cognitive Decline in the Elderly (IQCODE) - short form (Jorm, 1994) to identify and exclude patients with pre-stroke dementia (IQCODE score $>3$ ). The study was carried out in accordance with the Declaration of Helsinki (The Code of Ethics of the World Medical Association), and approved by the Ethical Committee of the Medical Faculty of the University of Nis, Republic of Serbia (No 12-98082/1). All the participants gave informed consent.

\subsection{EEG data acquisition}

EEG was recorded on the day of assessment, both in the subacute and the chronic stages of stroke, using a 19 channel NK-9100K EEG system (Nihon Kohden, Japan). We used standard EEG electrode placement and positioning according to the 10-20 International System (Fp1, Fp2, F7, F3, F4, F8, T3, C3, C4, T4, T5, P3, P4, T6, O1, and O2). Recordings were referenced to $(\mathrm{C} 3+\mathrm{C} 4) / 2$, the physical reference of the NK-9100K EEG system, with the ground electrode placed on the forehead. After conventional amplification and filtering (high pass $0.1 \mathrm{~Hz}$, notch 
175 filter $50 \mathrm{~Hz}$ ) the analog signals were digitized (250/s), and recorded for approximately 15

176 minutes in a resting, awake state with eyes closed.

\section{2.3. EEG data analysis}

178

179

180

181

182

183

184

185

186

187

188

189

190

191

192

193
All the EEG recordings were visually inspected and discriminated using EEGLAB to eliminate any ocular, muscular, and other types of artefacts before further analysis. The final EEG signals, consisting of 10 minute artefact-free periods, were analyzed in MATLAB R2011a, using software originally developed in MATLAB 6.5 (Ciric et al., 2015, 2016; Kalauzi et al., 2012; Lazic et al., 2015; 2017; Petrovic et al., 2013a, 2013b, 2014; Saponjic et al., 2013). We particularly analyzed the EEGs derived from the four lateral frontal (F3, F7, F4, F8), and corresponding lateral posterior (P3, P4, T5, T6) electrodes (Schleiger et al., 2014). After additional filtering (1-50 Hz band pass filter), we applied FFT algorithm to each individual recording, using a 10 second non-overlapping moving window resulting in $0.1 \mathrm{~Hz}$ frequency resolution (60 10 second Fourier epochs per each electrode), to calculate the relative EEG amplitudes of all the conventional frequency bands $(\delta=1.1-4 \mathrm{~Hz} ; \theta=4.1-8 \mathrm{~Hz} ; \mathrm{a}=8.1-13 \mathrm{~Hz} ; \beta$ $=13.1-30 \mathrm{~Hz} ; \gamma=30.1-50 \mathrm{~Hz})$.

Following the concept of "individual alpha frequency" (Klimesch, 1999), for each EEG recording and each electrode we calculated the weighted average of the alpha frequency (aAVG), using the alpha amplitude as a weighting factor:

$$
\mathrm{aAVG}=\frac{\sum_{f}(A m p(f) x f)}{\sum_{f} A m p(f)}
$$


194 where $f$ is a specific frequency ranging across the alpha frequency band and $A m p(f)$ is the 195 amplitude at $f$ (Hooper, 2005).

196 In addition, to analyze the EEG amplitude changes (the EEG microstructure), we 197 calculated the group probability density distributions of all the conventional EEG frequency band 198 relative amplitudes using the Probability Density Estimate (PDE) routine supplied with 199 MATLAB R2011a. As in our previous studies, in order to eliminate any influence from absolute 200 signal amplitude variations on the recordings, we calculated the relative Fourier amplitudes 201 (Ciric et al., 2015, 2016; Lazic et al., 2015; 2017; Petrovic et al., 2013a, 2013b, 2014; Saponjic 202 et al., 2013):

203

$$
(R A)_{b}=\frac{\sum_{b} A m p}{\sum_{t o t} A m p}, b=\{\delta, \theta, \alpha, \beta, \gamma\}
$$

204 For each electrode and each frequency band, PDE analysis was performed on the ensembles of 205 relative amplitudes by pooling the measured values from all subjects belonging to the corresponding group (the control, the subacute stage of the stroke or the chronic stage of the stroke). For the statistical analysis of PDEs the relative amplitude means were calculated for each 2 minutes.

We also calculated the inter-hemispheric and intra-hemispheric coherences by using the 210 "mscohere" routine of the MATLAB R2011a Signal Processing Toolbox, which computes the magnitude squared coherence between the signals $x$ and $y$ as:

$$
C_{(x y)}(f)=\frac{\left|P_{x y}(f)\right|^{2}}{P_{x x}(f) P_{y y}(f)}
$$


213 where $P_{x y}(f)$ is the cross spectrum of $x$ and $y$, while $P_{x x}(f)$ and $P_{y y}(f)$ denote the power

214 spectra of the two signals. For this purpose, the individual EEG signals, derived from the 215 corresponding electrodes, were concatenated and pooled within each group. For each electrode 216 pair, a coherence value of between 0 and 1 was calculated for every 10 seconds and each 217 frequency point (at a $0.1 \mathrm{~Hz}$ frequency resolution), within the overall $0.1-50 \mathrm{~Hz}$ range. Then the 218 values within each conventional frequency band were averaged for each spectrum, and their 219 means were calculated from the collection of all the available spectra. We calculated the group 220 mean coherence values for each 2 minutes, for each frequency band, each pair of electrodes, and 221 each group.

We also analyzed the topographic distribution of the alpha carrier frequency phase potentials (PPs) through changes in their carrier frequency phase shifts (PSs; Kalauzi et al., 2009; 2012). For this particular analysis, the individual EEG signals, derived from all 19 electrodes, were re-referenced offline to the common average reference (the average of all 19 electrodes). To be specific, for each pair of channels, their EEG recordings were treated as signals with variable amplitudes, but with the same alpha frequency (model details can be found in Kalauzi et al., 2012, Appendix B). Since alpha frequency is expected to be different between individuals ("individual alpha frequency“, Klimesch, 1999), the alpha frequency range limits used for PP analysis were determined individually, based on the previously determined aAVG, following the natural limits of the alpha peak position within each spectrum. For each subject and each pair of electrodes we calculated an alpha carrier frequency PS and alpha carrier frequency PP derived from the corresponding Fourier component PSs. Finally, we calculated the group alpha carrier frequency PPs (for the control, the subacute stage of the stroke and the 
236 subacute stage of the stroke vs. the control, the chronic stage of the stroke vs. the control, and the

237 chronic stage of stroke vs. the subacute stage of stroke) for each electrode. All the calculations

238 (the alpha carrier frequency PSs, the alpha carrier frequency PPs, the group mean alpha carrier

239 frequency PPs and the group alpha carrier frequency PP differences) were done using angular

240 arithmetic (Kalauzi et al., 2012, Appendix A, C, E). Since alpha carrier frequency PP is an

241 integral phase measure of a particular EEG channel in the alpha range, incorporating all alpha

242 Fourier components, it is possible to compare and subtract carrier frequency PP values even if

243 the aAVG frequency differs between groups, as was the case in this study. The final results of PP

244 analysis are presented as circles over corresponding electrodes, with diameters (small or large)

245 referring to the PP absolute values, and line types (solid or dashed) referring to the PP signs

246 (positive or negative). To be clear, since the PP calculation is based on angular variables, small

247 circles correspond to abs $(\mathrm{PP}) \approx 0^{\circ}$, while large circles correspond to abs $(\mathrm{PP}) \approx \pm 180^{\circ}$ (with a

248 solid line for positive and a dashed line for negative PP values). Therefore, due to their angular

249 nature, the large diameter circles represent similar PP values, regardless of the sign (e.g. PP =

$250178^{\circ}$ and $\mathrm{PP}=-179^{\circ}$ should be treated as close to each other since their actual difference is not

$251357^{\circ}$, but rather $3^{\circ}$ ). In this way all channels can be organized in a series (order) based on their

252 PP values: those channels leading in phase to all others will have the highest (large positive) PP

253 values, while the channels with the lowest (large negative) PP values would be the one following

254 all the others (Kalauzi et al., 2012). Based on these results, we have also drawn a topographic

255 pattern of "alpha flow" in the control group, and the subacute and chronic stage of the stroke, by

256 following the phase potential gradient (from the highest to the lowest PP values).

\section{2.4. Statistical analysis}


All the values are presented as means \pm SDs. The statistical analysis was done using the

259

260

261

262

263

264

265

266

267

268

269

270

271

272

273

274

275

276

277

Kruskal-Wallis ANOVA with a post-hoc Mann-Whitney U two-tailed test, and Spearman's correlation. In all cases the difference was considered significant for $\mathrm{p} \leq 0.05$.

\section{Results}

\subsection{Clinical and demographic assessments}

Table 1 summarizes the relevant demographic and clinical data of the control group and the stroke patients. There were 6 male and 4 female subjects among the stroke patients and 5 male and 6 female subjects in the control group. The age difference between stroke patients $(63.80 \pm 7.00$ in the subacute stage and $63.89 \pm 6.83$ in the chronic stage) and the healthy controls $(60.64 \pm 9.91)$ was not statistically significant $(z \geq-0.78 ; p \geq 0.44)$. There was no statistically significant difference in the years of education between the two groups $(z=-1.52 ; p$ $=0.13)$. The average NIHSS was $8.20 \pm 3.05$ on admission, $2.50 \pm 0.85$ at the time of the first assessment, and $2.11 \pm 0.78$ at a time of the follow-up assessment.

All the patients in the stroke group had their stroke in the vascular territory of the right MCA. The average lesion volume measured by the $\mathrm{ABC} / 2$ method was $9.34 \pm 6.49 \mathrm{~cm}^{3}$. The ischemic stroke lesion volumes and the affected structures for each patient are shown in Table 2.

As indicated in Table 1, MoCA scores in stroke patients during the subacute stage were significantly lower than in the healthy controls $(20.80 \pm 5.32$ vs. $25.64 \pm 3.20 ; \mathrm{z}=-2.12 ; \mathrm{p}=$ 0.03), by contrast with the chronic stage of the stroke where these MoCA score differences were 
278 not statistically significant in stroke patients vs. controls $(23.44 \pm 4.98$ vs. $25.64 \pm 3.20 ; \mathrm{z}=-$

$2790.88 ; \mathrm{p}=0.38)$

280

In addition, there was a significant difference between the subacute stroke patients and

281

282

283

284

285

286

287

the control group concerning MoCA-MIS (Table 1, $8.80 \pm 3.52$ vs. $11.91 \pm 2.98 ; \mathrm{z}=-1.98 ; \mathrm{p}=$ 0.05), whereas in the chronic stroke patients, the MoCA-MIS score returned to the control values (Table 1, $10.22 \pm 3.35$ vs. $11.91 \pm 2.98 ; \mathrm{z}=-1.15 ; \mathrm{p}=0.25$ ).

\subsection{EEG alpha activity following a minor stroke}

For all the analyzed frontal and posterior EEGs, the group mean relative amplitude EEG spectra showed prominent amplitude peaks in the alpha frequency range in all groups, but with the alpha peaks shifted toward lower frequencies in stroke patients, particularly in the subacute stage (Fig. 1). Further analysis of $\alpha \mathrm{AVG}$ frequency revealed a generalized decrease in $\alpha \mathrm{AVG}$ following a stroke. In particular, and in contrast to the aAVG in the control group that was in the range of $10.38-10.54 \mathrm{~Hz}$, the subacute stroke group had an $\alpha \mathrm{AVG}$ in the range of 10.11-10.23 $\mathrm{Hz}$, whereas the chronic stroke group had an $\alpha \mathrm{AVG}$ in the range of $10.22-10.38 \mathrm{~Hz}$.

A statistically significant slower alpha frequency in stroke patients vs. controls was demonstrated only for the subacute stage at F3, F4, F7, F8, P3 and P4 $\left(\chi^{2} \geq 6.11 ; \mathrm{p} \leq 0.05 ; \mathrm{z} \geq\right.$ 3.32; $\mathrm{p} \leq 0.03)$, but with no change in the $\alpha \mathrm{AVG}$ at T5 and T6 $\left(\chi^{2} \geq 3.09 ; \mathrm{p} \geq 0.18 ; \mathrm{z} \geq-1.66 ; \mathrm{p} \geq\right.$ 0.10). However, all the stroke induced alpha frequency changes returned to the control values ( $\mathrm{z}$ $\geq-1.90 ; p \geq 0.06)$ in the chronic stage.

In our study we did not demonstrate the amplitude change of the slower alpha in subacute and chronic stroke patients vs. controls $\left(\chi^{2} \geq 1.44 ; \mathrm{p} \geq 0.14 ; \mathrm{z} \geq-1.72 ; \mathrm{p} \geq 0.09 ;\right.$ data not shown), but we have shown the delta, theta, and beta amplitude alterations (Fig. $2 ; \chi^{2} \geq 6.61 ; p \leq 0.04$ ). 
300 The frontal cortex exhibited a general beta amplitude decrease in both subacute and chronic

301 stroke patients vs. controls (Fig. 2A, beta; $\chi^{2} \geq 15.21 ; p=10^{-4} ; \mathrm{z} \geq-4.08 ; \mathrm{p} \leq 0.01$ ). This beta

302 amplitude decrease was followed by a long-lasting theta amplitude increase across all the frontal

303 and posterior channels (Fig. 2A, B, theta; $\chi^{2} \geq 7.86 ; p \leq 0.02 ; z \geq-5.64 ; p \leq 0.03$ ), with the

304 exception of F8 and T5 where the theta amplitude returned to the control values in the chronic

305 stage $(\mathrm{z} \geq-1.50 ; \mathrm{p} \geq 0.13)$. Delta amplitude alterations were in evidence only in the posterior

306 channels (Fig. 2B, delta; $\chi^{2} \geq 6.61 ; \mathrm{p} \leq 0.04$ ): the delta amplitude increased at P3 in the chronic

307 stage of stroke patients vs. controls $(\mathrm{z}=-2.42 ; \mathrm{p}=0.02)$ in contrast to a transient increase at T5

308 and T6 in the subacute stage $(\mathrm{z} \geq-2.90 ; \mathrm{p} \leq 0.01)$.

309

310

311

312

313

314

315

316

317

318

319

320

\subsection{Inter-hemispheric and intra-hemispheric coherences following a minor stroke}

Inter-hemispheric coherence analysis between the frontal electrodes, in both subacute and chronic stroke patients, revealed significantly increased coherences in the alpha frequency range in contrast to the control group (Fig. 3A, B; $\chi^{2} \geq 6.56 ; \mathrm{p} \leq 0.04 ; \mathrm{z} \geq-3.24 ; \mathrm{p} \leq 0.02$ ). This sustainably increased synchronization of slower alpha, caused by the stroke, was particularly expressed at the F3-F4 electrodes, and co-localized with the underlying neuropathology of the ischemic stroke (see Table 2). Conversely, the posterior electrodes expressed a generalized longlasting desynchronization in all the frequency ranges (Fig. $3 \mathbf{C}, \mathbf{D} ; \chi^{2} \geq 6.87 ; \mathrm{p} \leq 0.03 ; \mathrm{z} \geq-4.82$; $\mathrm{p} \leq 0.04)$, but with no change in alpha coherences $\left(\chi^{2} \geq 2.59 ; \mathrm{p} \geq 0.14 ; \mathrm{z} \geq 10.63 ; \mathrm{p} \geq 0.10\right)$ in either subacute or chronic stroke patients vs. the controls.

In addition, in the chronic stage we demonstrated increased theta and beta synchronizations at the F3-F4 electrodes (Fig. 3A; $\chi^{2} \geq 7.67 ; \mathrm{p} \leq 0.02 ; \mathrm{z} \geq-2.84 ; \mathrm{p} \leq 0.02$ ) 
321 alongside broad spectrum (delta, theta, beta and gamma) desynchronization at the frontal F7-F8

322 electrodes (Fig. 3B; $\chi^{2} \geq 6.59 ; \mathrm{p} \leq 0.04 ; \mathrm{z} \geq-3.00 ; \mathrm{p} \leq 0.05$ ).

324 shown within the ipsi-lesional hemisphere (Fig. 4D-F; $\left.\chi^{2} \geq 6.06 ; p \leq 0.05\right)$. We demonstrated

325 sustainably increased alpha coherences across the F4-F8, F4-T6 and F4-P4 electrodes during

326 both stages of stroke vs. the controls $(\mathrm{z} \geq-5.11 ; \mathrm{p} \leq 0.04)$. In addition, there were also

327 inconsistent alterations in the delta, theta, beta and gamma coherences within the ipsi-lesional

328 hemisphere in the subacute and chronic stages of stroke patients vs. the controls (Fig. 4D-F; $\chi^{2} \geq$

$6.84 ; \mathrm{p} \leq 0.03 ; \mathrm{z} \geq-4.24 ; \mathrm{p} \leq 0.02)$. Conversely, the contra-lesional hemisphere demonstrated an increased synchronization in the theta frequency range, but only in subacute stroke patients (Fig.

4A-C; $\left.\chi^{2} \geq 6.49 ; \mathrm{p} \leq 0.04 ; \mathrm{z} \geq-4.51 ; \mathrm{p} \leq 0.05\right)$. This transient theta coherence augmentation was followed by inconsistent delta, alpha, beta and gamma coherence alterations in both the subacute and chronic stroke stages vs. the controls (Fig. 4A-C; $\chi^{2} \geq 9.08 ; \mathrm{p} \leq 0.01 ; \mathrm{z} \geq-4.86 ; \mathrm{p} \leq 0.03$ ).

\subsection{Topography of $\alpha A V G$ phase potentials following a minor stroke} stroke patients, and the alpha in the chronic stroke patients and healthy controls (Fig. 1) with their corresponding PP differences are depicted in Fig. 5. We have shown that the frontal cortex of the stroke patients vs. the controls expressed the greatest and long-lasting PP differences (Fig. 5A, B). This long-term impact of the stroke on the alpha PP differences was additionally confirmed by the almost non-existing PP differences between the subacute and chronic stage of the stroke patients (Fig. 5C). In addition, whereas the control group of patients showed relatively 
343 Subacute Stroke) and the chronic stroke group of patients (Fig. 5B, Chronic Stroke)

344 demonstrated a clear antero-posterior difference in alpha PPs, with the smallest frontal PPs vs.

345 the largest posterior PPs. Consequently, the great majority of channels over the frontal cortex

346 exhibited negative PP differences (dashed circles, Fig. 5A, Subacute Stroke vs. Control; Fig.

347 5B, Chronic Stroke vs. Control), meaning that the frontal alpha waves were more delayed in

348 the stroke patients than in the controls, compared to other channels. We identified the greatest

349 decrease of PPs over the frontal cortex at F4 (Fig. 5A, Subacute Stroke vs. Control; Fig. 5B,

350 Chronic Stroke vs. Control) and during both stages of stroke, which, as a sign of the largest

351 delay in the newly emerged slower alpha, might be related to the confirmed neuropathology of

352 an ischemic stroke.

Another way to present the group mean alpha PPs is to draw them as vectors in a unit circle (Fig. 6A). In cases where the PPs of all channels are closely distributed in a unit circle, forming "a bundle", this formation could be considered as having a "source" (the channel with the highest PP, where the alpha wave is first recorded) or "drain" (the channel with the lowest PP, where the alpha wave is last recorded). Since all the demonstrated alpha PPs were distributed across the whole circle, rather than being concentrated within "a bundle", the unit circle could be considered only as a closed loop or rather not a "source" or "drain" unit circle. Therefore, we have drawn a topographic pattern of the "alpha flow" for the control group (Fig. 6B Control), the subacute stroke group (Fig. 6B, Subacute Stroke), and the chronic stroke group (Fig. 6B, descending PP gradient. The greatest alterations in the "alpha flow" in stroke patients vs. the controls were demonstrated within the frontal cortex (Fig. 6C, indicated by solid red arrows), with F4 undergoing the most intensive PP change, leading to the reorganization of the PP 
366 channel sequence. To be specific, in control group the F4 PP neighbors were positioned

367 longitudinally in the same ipsi-lateral hemisphere (Fig. 6C, Control), whereas in the subacute

368 (Fig. 6C, Subacute Stroke) and chronic stage of the stroke patients (Fig. 6C, Chronic Stroke)

369 F4 was included in the newly established antero-posterior inter-hemispheric spiral PP pattern,

370 indicating that the stroke had induced a sustained augmentation of inter-hemispheric interactions.

\subsection{Correlations of the average EEG alpha frequency and cognitive functions} aAVG are presented in Table 3 and Fig. 7. We did not find a significant correlation between the

While the MoCA scores in our 10 subacute stroke patients were significantly correlated with the aAVGs at F4 $(\rho=0.74, p=0.02), \mathrm{P} 3(\rho=0.65, p=0.04), \mathrm{P} 4(\rho=0.77, p=0.01)$ and T6 $(\rho=0.74, p=0.02)$, the MoCA-MIS scores were not significantly correlated with the $\alpha$ AVGs at any EEG channel $(\mathrm{p} \geq 0.09)$.

Although the correlation between the MoCA scores and the aAVGs in our 9 chronic stroke patients was not significant (they returned to the control correlation values, $p \geq 0.38$ ), the MoCA-MIS became significantly correlated with their $\alpha$ AVGs at P3 $(\rho=0.72, p=0.03)$, P4 $(\rho=$

0.92, $\mathrm{p}<0.01)$, T5 $(\rho=0.77, \mathrm{p}=0.02)$ and T6 $(\rho=0.92, \mathrm{p}<0.01)$.

\section{Discussion}


neuropathology, re-organizational processes, and cognitive functions during the subacute and slower alpha frequency $(\alpha \mathrm{AVG})$ in the subacute stroke patients vs. controls in a small sample of

highly synchronized intra-hemispherically, overlying the ipsi-lesional hemisphere (Fig. 4), and inter-hemispherically, overlying the frontal cortex (Fig. 3). In addition, the disturbances of EEG alpha activity in subacute stroke patients vs. controls were expressed as a decrease in alpha PPs over the frontal cortex (indicating the delay of the slower alpha), and an altered "alpha flow", indicating the sustained augmentation of inter-hemispheric interactions (Figs. 5, 6). Although the stroke induced alpha frequency changes (the slower alpha) returned to the control values in

lesional hemisphere, the increased alpha F3-F4 inter-hemispheric synchronization, the delayed 399 alpha waves, and the newly established inter-hemispheric "alpha flow" within the frontal cortex, remained as a permanent consequence of the minor stroke.

One way to consider the sustained alterations in EEG alpha activity in minor stroke 402 patients is as a compensatory brain response contributing to functional recovery. It could be assumed that these re-organizational changes, expressed as a permanently established inter404 hemispheric "alpha flow", may result from unbalanced inter-hemispheric inhibition, a 405 phenomenon well known from transcranial magnetic stimulation (TMS) studies assessing motor

406 function recovery after hemiparetic stroke (Büterfisch et al., 2008; Manganotti et al., 2008; 407 Shimizu et al., 2002). To specify, normal brain activity depends on the balance between 408 excitatory and inhibitory signaling, and following a stroke this balance is shifted toward 
409 excitation, leading to a disturbance in competitive inhibition between the two hemispheres,

410 which is normally maintained during activation. The unilateral brain lesions reduce inhibition

411 from the affected (ipsi-lesional) to the contralateral (contra-lesional) hemisphere, which in turn

412 becomes hyperactive and potentiates the inhibition of the ipsi-lesional hemisphere (Büterfisch et 413 al., 2008). In addition, evidence of brain hyperexcitability is provided by studies in animal 414 models of stoke, demonstrating the down-regulation of the GABA receptor function and up415 regulation of the NMDA receptor function (Que et al., 1999; Redecker et al., 2002). The 416 increased excitability of the motor cortex and dynamical alterations to inter-hemispheric 417 inhibition in post-stroke patients, demonstrated by the paired-pulse TMS technique, have been 418 related to the degree of clinical motor recovery (Büterfisch et al., 2008; Manganotti et al., 2008). established inter-hemispheric "alpha flow" in our study could be a way for the brain to compensate for the lesion and restore the lost function. disturbance of the remote functional networks. PET and fMRI imaging studies (Carter et al., 2010; He et al., 2007; Warren et al., 2009), investigating neural interactions between the brain regions in stroke patients, have demonstrated the disruption of the functional connectivity between the affected hemisphere and the rest of the brain. This observed impairment of neuronal interactions reflects neurological deficits (Carter et al., 2010; He et al., 2007; Warren et al., 428 2009). Moreover, MEG and EEG studies have shown the disruption of the functional 429 connectivity of specific frequency bands, such as decreased alpha band synchronization 430 (coherence) between the affected region and the rest of the brain (Dubovik et al., 2012; Westlake 431 et al., 2012). The magnitude of alpha band synchronization was negatively correlated with the 
432 functional impairment, with a distinct increase in alpha synchronization during recovery, and in

433 parallel with the improvement of the neurological deficits (Westlake et al., 2012).

434

435

436

437

438

439

440

441

442

443

444

445

446

447

448

449

450

451

452

454

453 Our results of the cognitive assessments demonstrated the decreased MoCA and MoCA-

In our study, the functional connectivity between the brain regions was estimated using EEG coherence, particularly the mean coherence between the electrodes overlaying the area of the confirmed neuropathology. We have demonstrated increased alpha synchronization between the ipsi-lesional and contra-lesional frontal cortex (Fig. 3), and within the overall ipsi-lesional hemisphere (Fig. 4). This generalized alpha synchronization indicates the compensatory recruitment of peri-lesional as well as contra-lesional brain areas during post-stroke recovery.

We should mention here that the reference technique we used was determined by the manufacturer of the EEG apparatus (NK-9100K EEG system Nihon Kohden, Japan), and it was a limitation of our study. However, although the reference technique and volume conduction could affect coherence measures, we demonstrated an increased alpha synchronization, both interhemisphericaly, overlaying the frontal cortex (Fig. 3) and intra-hemisphericaly, overlaying the ipsi-lesional hemisphere (Fig. 4) in the stroke patients vs. the controls. In addition, our coherence analysis also included other frequency bands, and not all of them were increased in the stroke patients vs. the controls. Conversely, many of them exhibited desynchronization, i.e. decreased coherence (Fig. 3, Fig. 4). If we had made a mistake, and our coherence measures had been affected by volume conduction, than the error would be incorporated in all the coherence measures, and all the experimental groups due to the reference impact. Despite that, we still showed the differences in alpha (and other frequency bands) coherences between stroke patients and healthy controls. MIS scores in stroke patients vs. the controls, but only during the subacute stage, indicating only 
455 a transient cognitive impairment due to a minor stroke. In parallel with the observed cognitive

456 dysfunction, the stroke patients also expressed a transient decrease in aAVG (Fig. 1). In

457 addition, our study revealed significant correlations between $\alpha \mathrm{AVG}$ and the cognitive assessment

458 scores in stroke patients vs. the controls (Table 3; Fig. 7). In contrast to the controls, who

459 showed no correlations, the $\alpha \mathrm{AVG}$ in stroke patients were positively correlated with MoCA

460 scores only during the subacute stage of the stroke, whereas MoCA-MIS scores became

461 significantly correlated with aAVGs during the chronic stage of the stroke.

Generally, the greatest behavioral recovery following a stroke is shown within the first 4

weeks, but the functional recovery may continue beyond this initial period, particularly when

restorative therapies are included during rehabilitation (Green, 2003; Grefkes and Ward, 2014;

Teasell et al., 2005). However, the rate of overall recovery depends on the severity of the stroke and the domain affected, with mild initial impairments showing faster improvement and better

467 final outcomes in contrast to more severe strokes (Cramer, 2008). When it comes to cognitive 468 impairment, most stroke survivors show their maximum recovery within the first 3 months 469 (Desmond et al., 1996). Our results are in accordance with the concept of spontaneous functional 470 recovery following a stroke. All the subjects in our study were assessed as minor stroke patients

471 (NIHSS < 4) with transient cognitive impairments that were demonstrated only during the 472 subacute stage. Although the MoCA-MIS scores along with aAVGs returned to the control 473 values in the chronic stroke patients, they became highly correlated (functionally coupled, Table 474 3, Fig. 7), only over the posterior brain region. This newly established functional coupling 475 (topography) could be a compensatory reorganization following an ischemic lesion.

The functional relevance of alpha EEG oscillations has been extensively studied 477 (Klimesch, 1999, 2012; Palva and Palva, 2007). A lot of evidence suggests that alpha oscillations 
478 reflect cognitive and memory performance (Klimesch, 1999). A study by Patten et al., (2012) has

479 shown that the properties of alpha wave propagation over the scalp correlate with the speed of 480 information processing, particularly for alpha waves traveling in the frontal-to-occipital 481 direction. On the other hand, reaction time, as a measure of information processing speed, is 482 correlated with alpha frequency: a lower alpha frequency means a slower reaction time, hence 483 poor cognitive and memory performance (Klimesch, 1999). Moreover, the propagation of 484 electrical oscillations can be affected by brain pathologies, such as tumors, ischemia or 485 vasogenic edema (Jochmann et al., 2011). Based on these findings, we can speculate that the 486 post-stroke cognitive impairment, as well as the alpha frequency decrease, demonstrated in our 487 study, could be a consequence of the altered propagation of alpha waves due to a stroke induced 488 lesion.

In addition, the alpha frequency is known to vary with age (increasing up to adulthood and decreasing in older age), age related diseases, or even a lack of school education (Klimesch, 1999). However, no statistically significant differences in age or education between the control 492 and stroke group of patients and the lack of (other) neurological and psychiatric diseases in the stroke patients, indicates a stroke induced slower alpha frequency.

Apart from the EEG alpha activity, we have shown alterations to other frequency bands, 495 and as expected, the stroke patients demonstrated pathological EEG slowing during both stages of a minor stroke (Fig. 2). Although, the stroke induced slowing of EEG is well documented 497 (Burghaus et al., 2013; Finnigan et al., 2016; Jordan, 2004), in our study the alpha slowing was 498 followed by theta amplitude augmentation and beta amplitude attenuation, particularly over the 499 frontal cortex. In addition, the minor stroke induced long-term alpha inter-hemispheric 
500 synchronization within the frontal cortex, followed by permanent delta, theta, beta, and gamma 501 inter-hemispheric desynchronization within the parietal and temporal cortex (Fig. 3).

503 specific sample of stroke patients ( 9 stroke patients), all with minor strokes (NIHSS $<4$ ) in the 504 distribution of the right MCA. Although our findings could benefit from a larger sample size, we 505 still can not assume that our results might be extrapolated to other types of stroke patients or 506 even to left MCA stroke cases. Future research including other types of stroke patients (and a 507 larger sample size) could resolve this issue and further prove or disprove the potential value of 508 our EEG measurements as general qEEG indices.

Our study of a small sample of right MCA stroke patients has demonstrated EEG alpha 510 activity alterations following a minor stroke. Whereas the transient slowing of the alpha

511 frequency was related to cognitive impairment in subacute stroke patients, the sustainably 512 increased alpha synchronization, delayed alpha waves, and a newly established frontal inter513 hemispheric "alpha flow" were permanent consequences. These permanent alpha alterations 514 revealed the "hidden" stroke neuropathology or post-stroke re-organizational processes, despite 515 the fact that the cognitive impairment returned to the control value. Our study indicates that 516 slower EEG alpha generation, synchronization and "flow" may potentially serve as biomarkers 517 of cognitive impairment onset and/or post-stroke compensatory re-organizational processes. 


\section{References}

520

521 Borich MR, Brown KE, Lakhani B, Boyd LA. Applications of electroencephalography to

522 characterize brain activity: Perspectives in stroke. J Neurol Phys Ther 2015; 39: 43-51.

524 Burghaus L, Liu WC, Dohmen C, Haupt WF, Fink GR, Eggers C. Prognostic value of 525 electroencephalography and evoked potentials in the early course of malignant middle cerebral 526 artery infarction. Neurol Sci 2013; 34: 671-678.

527

528 Bütefisch CM, Wessling M, Netz J, Seitz RJ, Hömberg V. Relationship between 529 interhemispheric inhibition and motor cortex excitability in subacute stroke patients. 530 Neurorehabil Neural Repair 2008; 22: 4-21.

531

532 Carter AR, Astafiev SV, Lang CE, Connor LT, Rengachary J, Strube MJ, Pope DL, Shulman

533 GL, Corbetta M. Resting interhemispheric functional magnetic resonance imaging connectivity 534 predicts performance after stroke. Ann Neurol 2010; 67: 365-75.

536 Ciric J, Lazic K, Petrovic J, Kalauzi A, Saponjic J. Age-related disorders of sleep and motor 537 control in the rat models of functionally distinct cholinergic neuropathology. Behav Brain Res $5382016 ; 301: 273-286$. 
539 Ciric J, Lazic K, Petrovic J, Kalauzi A, Saponjic J. Aging induced cortical drive alterations

540 during sleep in rats. Mech Ageing Dev 2015; 146-148: 12-22.

541

542 Cramer SC. Repairing the human brain after stroke: I Mechanisms of spontaneous recovery. Ann

543 Neurol 2008; 63: 272-287.

Desmond DW, Moroney JT, Sano M, Stern Y. Recovery of Cognitive Function After Stroke.

546 Stroke 1996; 27: 1798-1803.

547

548 Dubovik S, Pignat JM, Ptak R, Aboulafia T, Allet L, Gillabert N, Magnin C, Albert F, Momjian-

549 Mayor I, Nahum L, Lascano AM, Michel CM, Schnider A, Guggisberg AG. The behavioral 550 significance of coherent resting-state oscillations after stroke. Neuroimage 2012; 61: 249-257.

Dubovik S, Ptak R, Aboulafia T, Magnin C, Gillabert N, Allet L, Pignat JM, Schnider A, Guggisberg AG. EEG alpha band synchrony predicts cognitive and motor performance in patients with ischemic stroke. Behav Neurol 2013; 26: 187-189.

556 Finnigan S, Rose SE, Chalk JB. Rapid EEG changes indicate reperfusion after tissue 557 plasminogen activator injection in acute ischaemic stroke. Clin Neurophysiol 2006; 117: 23385582339. 
559 Finnigan S, van Putten MJAM. EEG in ischaemic stroke: Quantitative EEG can uniquely inform

560 (sub-)acute prognoses and clinical management. Clin Neurophysiol 2013; 124: 10-19.

561

562 Finnigan S, Wong A, Read S. Defining abnormal slow EEG activity in acute ischaemic stroke:

563 Delta/alpha ratio as an optimal QEEG index. Clin Neurophysiol 2016; 127: 1452-1459.

Finnigan SP, Rose SE, Walsh M, Griffin M, Janke AL, McMahon KL, Gillies R, Strudwick

MW, Pettigrew CM, Semple J, Brown J, Brown P, Chalk JB. Correlation of quantitative EEG in acute ischemic stroke with 30-day NIHSS score: comparison with diffusion and perfusion MRI. Stroke 2004; 35: 899-903.

Folstein MF, Folstein SE, McHugh PR. Mini-mental state. A practical method for grading cognitive state of patients for clinician. J Psychiatr Res 1975; 12: 189-198.

Foreman B, Claassen J. Quantitative EEG for the detection of brain ischemia. In: Vincent JL,

574 editor. Annual Update in Intensive Care and Emergency Medicine. Berlin Heidelberg: Springer575 Verlag; 2012. p. 746-758.

576

577 George PM, Steinberg GK. Novel stroke therapeutics: Unraveling stroke pathophysiology and its 578 impact on clinical treatments. Neuron 2015; 87: 297-309. 
579 Green JB. Brain reorganization after stroke. Top Stroke Rehabil 2003; 10: 1-20.

580

581 Grefkes C, Ward NS. Cortical reorganization after stroke: How much and how functional?

582 Neuroscientist 2014; 20: 56-70.

583

584 He BJ, Snyder AZ, Vincent JL, Epstein A, Shulman GL, Corbetta M. Breakdown of functional 585 connectivity in frontoparietal networks underlies behavioral deficits in spatial neglect. Neuron 586 2007; 53: 905-18.

587

588 Hooper GS. Comparison of the distributions of classical and adaptively aligned EEG power 589 spectra. Int J Psychophysiol 2005; 55: 179- 189.

590

591 Hossmann KA. Viability thresholds and the penumbra of focal ischemia. Ann Neurol 1994; 36, $592 \quad 557-565$.

593

594 Iyer KK. (in press) Effective assessments of electroencephalography during stroke recovery: 595 contemporary approaches and considerations. J Neurophysiol 2017; DOI: 596 10.1152/jn.00206.2017. 
598 Jochmann T, Güllmar D, Haueisen J, Reichenbach JR. Influence of tissue conductivity changes 599 on the EEG signal in the human brain: a simulation study. Z Med Phys 2011; 21: 102-112.

600

601 Jokinen H, Melkas S, Ylikoski R, Pohjasvaara T, Kaste M, Erkinjuntti T, Hietanen M. Post602 stroke cognitive impairment is common even after successful clinical recovery. Eur J Neurol $6032015 ; 22: 1288-1294$.

604

605 Jordan KG. Emergency EEG and continuous EEG monitoring in acute ischemic stroke. J Clin 606 Neurophysiol 2004; 21: 341-352.

607

Jorm A F. A short form of the Informant Questionnaire on Cognitive Decline in the Elderly 609 (IQCODE): development and cross-validation. Psychol Med 1994; 24: 145-153.

610

611 Julayanont P, Brousseau M, Chertkow H, Phillips N, Nasreddine ZS. Montreal Cognitive 612 Assessment Memory Index Score (MoCA-MIS) as a predictor of conversion from mild cognitive 613 impairment to Alzheimer's disease. J Am Geriatr Soc 2014; 62: 679-684.

614

615 Kalaria RN, Akinyemi R, Ihara M. Stroke injury, cognitive impairment and vascular dementia. 616 Biochim Biophys Acta 2016; 1862: 915-925. 
617 Kalauzi A, Kesic S, Saponjic J. Cortico-pontine theta synchronization phase shift following 618 monoaminergic lesion in rat. J Physiol Pharmacol 2009; 60: 79-84.

619

620 Kalauzi A, Vuckovic A, Bojić T. EEG alpha phase shifts during transition from wakefulness to 621 drowsiness. Int J Psychophysiol 2012; 86: 195-205.

622

623 Klimesch W. EEG alpha and theta oscillations reflect cognitive and memory performance: a 624 review and analysis. Brain Res Rev 1999; 29: 169-195.

625

626 Klimesch W. Alpha-band oscillations, attention, and controlled access to stored information. 627 Trends Cogn Sci 2012; 16: 606-617.

628

629 Lazic K, Petrovic J, Ciric J, Kalauzi A, Saponjic J. Impact of anesthetic regimen on the 630 respiratory pattern, EEG microstructure and sleep in the rat model of cholinergic Parkinson's 631 disease neuropathology. Neuroscience 2015; 304: 1-13.

632

633 Lazic K, Petrovic J, Ciric J, Kalauzi A, Saponjic J. REM sleep disorder following general 634 anesthesia in rat. Physiol Behav 2017; 168: 41-54.

635 
636 Manganotti P, Acler M, Zanette GP, Smania N, Fiaschi A. Motor cortical disinhibition during 637 early and late recovery after stroke. Neurorehabil Neural Repair 2008; 22: 396-403.

638

639 Mijajlović MD, Pavlović A, Brainin M, Heiss WD, Quinn TJ, Ihle-Hansen HB, Hermann DM, 640 Assayag EB, Richard E, Thiel A, Kliper E, Shin YI, Kim YH, Choi SH, Jung S, Lee YB, 641 Sinanović O, Levine DA, Schlesinger I, Mead G, Milošević V, Leys D, Hagberg G, Ursin MH, 642 Teuschl Y, Prokopenko S, Mozheyko E, Bezdenezhnykh A, Matz K, Aleksić V, Muresanu D, 643 Korczyn AD, Bornstein N. Post-stroke dementia - a comprehensive review. BMC Med 2017; 15: 64411.

645

646 Nagata K, Tagawa K, Hiroi S, Shishido F, Uemura K. Electroencephalographic correlates of 647 blood flow and oxygen metabolism provided by positron emission tomography in patients with 648 cerebral infarction. Electroencephalogr Clin Neurophysiol 1989; 72: 16-30.

649

650 Nasreddine ZS, Phillips NA, Bedirian V, Charbonneau S, Whitehead V, Collin I, Cummings JL,

651 Chertkow H. The montreal cognitive assessment, MoCA: A brief screening tool for mild 652 cognitive impairment. J Am Geriatr Soc 2005; 53: 695-699.

653

654 Palva S, Palva JM. New vistas for alpha-frequency band oscillations. Trends Neurosci 2007; 30: 655 150-158. 
656 Patten TM, Rennie CJ, Robinson PA, Gong P. Human cortical traveling waves: dynamical 657 properties and correlations with responses. PLoS One 2012; 7: e38392.

658

659 Pendlebury ST, Rothwell PM. Prevalence, incidence, and factors associated with pre-stroke and 660 post-stroke dementia: a systematic review and meta-analysis. Lancet Neurol 2009; 8: 1006-1018.

661

662 Petrovic J, Ciric J, Lazic K, Kalauzi A, Saponjic J. Lesion of the pedunculopontine tegmental 663 nucleus in rat augments cortical activation and disturbs sleep/wake state transitions structure. 664 Exp Neurol 2013a; 247: 562-571.

665

Petrovic J, Lazic K, Ciric J, Kalauzi A, Saponjic J. Topography of the sleep/wake states related 667 EEG microstructure and transitions structure differentiates the functionally distinct cholinergic 668 innervation disorders in rat. Behav Brain Res 2013b; 256: 108-118.

669

670

Petrovic J, Lazic K, Kalauzi A, Saponjic J. REM sleep diversity following the pedunculopontine

671 tegmental nucleus lesion in rat. Behav Brain Res 2014; 271: 258-268.

672

673 Que M, Schiene K, Witte O, Zilles K. Widespread up-regulation of N-methyl-D-aspartate 674 receptors after focal photothrombotic lesion in rat brain. Neurosci Lett 1999; 273: 77-80. 
675 Redecker C, Wang W, Fritschy J, Witte O. Widespread and long-lasting alterations in 676 GABA(A)-receptor subtypes after focal cortical infarcts in rats: mediation by NMDA-dependent 677 processes. J Cereb Blood Flow Metab 2002; 22: 1463-1475.

678

679 Saponjic J, Petrovic J, Kalauzi A, Ciric J, Lazic K, Radulovacki M, Carley DW. Sleep-state 680 related EEG amplitude distribution in the rat model of cortical cholinergic innervation disorder. 681 Sleep Biol Rhythms 2013; 11: 105-115.

682 684 and screening for post-stroke cognitive deficits: The power of four electrodes. Int J 685 Psychophysiol 2014; 94: 19-24.

686 prognostication of cognitive impairment. Psychophysiol 2017; 54: 301-309. 
695 Sheorajpanday RVA, Nagels G, Weeren AJTM, De Deyn PP. Quantitative EEG in ischemic 696 stroke: Correlation with infarct volume and functional status in posterior circulation and lacunar 697 syndromes. Clin Neurophysiol 2011; 122: 884-890.

698

699 Shimizu T, Hosaki A, Hino T, Sato M, Komori T, Hirai S, Rossini PM. Motor cortical 700 disinhibition in the unaffected hemisphere after unilateral cortical stroke. Brain 2002; 125: 18967011907.

702

703

Song Y, Zang DW, Jin YY, Wang ZJ, Ni HY, Yin JZ, Ji DX. Background rhythm frequency and 704 theta power of quantitative EEG analysis: predictive biomarkers for cognitive impairment post705 cerebral infarcts. Clin EEG Neurosci 2015; 46: 142-146.

706

707

Sturm JW, Dewey HM, Donnan GA, Macdonell RAL, McNeil JJ, Thrift AG. Handicap after 708 stroke: How does it relate to disability, perception of recovery, and stroke subtype? The North East Melbourne stroke incidence study (NEMESIS). Stroke 2002; 33: 762-768.

710

711 Sun JH, Tan L, Yu JT. Post-stroke cognitive impairment: epidemiology, mechanisms and 712 management. Ann Transl Med 2014; 2: 1-16. 
714 Teasell R, Bayona NA, Bitensky J. Plasticity and reorganization of the brain post stroke. Top

715 Stroke Rehabil 2005; 12: 11-26.

716

717 Thiel A, Cechetto DF, Heiss WD, Hachinski V, Whitehead SN. Amyloid burden, 718 neuroinflammation, and links to cognitive decline after ischemic stroke. Stroke $2014 ; 45: 2825-$ 7192829.

720

721 Veale JF. Edinburgh Handedness Inventory - Short Form: a revised version based on 722 confirmatory factor analysis. Laterality 2014; 19: 164-177.

724 Warren JE, Crinion JT, Lambon Ralph MA, Wise RJ. Anterior temporal lobe connectivity 725 correlates with functional outcome after aphasic stroke. Brain 2009; 132: 3428-3442.

726

727 Westlake KP, Hinkley LB, Bucci M, Guggisberg AG, Byl N, Findlay AM, Henry RG, Nagarajan 728 SS. Resting state alpha-band functional connectivity and recovery after stroke. Exp Neurol 2012; 729 237: 160-169. 
731

732

733

734

735

736

737

738

739

740

741

742

743

744

745

746

747

748

749

750

751

752

753

\section{Figure Legends}

\section{Table 1. Demographic and clinical characteristics of stroke patients and healthy controls.}

Bold numbers indicate statistically significant values at $\mathrm{p} \leq 0.05$.

Table 2. Patient's stroke details (Stroke neuropathology: the volume of lesions and the brain structures affected).

Table 3. Correlations of MoCA and MoCA-MIS cognitive assessments and $\alpha A V G$ in the subacute and chronic stages of stroke patients vs. healthy controls.

Bold numbers indicate statistically significant correlations at $\mathrm{p} \leq 0.05$.

Suppl Table 1 for Fig. 3. The mean inter-hemispheric coherence in the subacute and chronic stages of stroke patients vs. healthy controls.

Bold numbers indicate statistically significant mean values at $\mathrm{p} \leq 0.05$.

Suppl Table 2 for Fig. 4. The mean intra-hemispheric coherence in the subacute and chronic stages of stroke patients vs. healthy controls.

Bold numbers indicate statistically significant mean values at $\mathrm{p} \leq 0.05$.

Fig. 1. Alterations in alpha frequency following a minor stroke. Group mean relative amplitude spectra of the frontal (A), parietal and temporal (B) EEG channels in the subacute $(\mathrm{n}=$ 10) and chronic $(n=9)$ stages of stroke patients vs. healthy controls $(n=11)$. 
754 We have demonstrated statistically significant slower alpha ( $\alpha$ AVG) at F3, F4, F7, F8, P3 and P4

755 in the subacute stroke patients vs. the controls $(\mathrm{p} \leq 0.03)$. In the chronic stage, all stroke induced

756 alpha frequency changes returned to the control values ( $p \geq 0.06)$.

757 Bold numbers and asterisks indicate a statistically significant decrease in $\alpha \mathrm{AVG}$ following a 758 stroke.

759

760

Fig. 2. EEG microstructure following a minor stroke. Group mean probability density

761

762

763

764

765

766

767

768

769

770

771

772

773

774

775

776 distributions (PDEs) of the delta, theta and beta relative amplitudes at the frontal (A), parietal and temporal $(\mathbf{B})$ EEG channels in the subacute $(\mathrm{n}=10)$ and chronic $(\mathrm{n}=9)$ stages of stroke patients vs. healthy controls $(\mathrm{n}=11)$.

The slower alpha in stroke patients with no amplitude change $(\mathrm{p} \geq 0.14)$ was followed by a generally augmented theta $(\mathrm{p} \leq 0.03)$, alongside an attenuated beta only within the frontal cortex and during both the subacute and chronic stage of the stroke $(\mathrm{p} \leq 0.01)$. These stroke induced theta and beta amplitude alterations were followed by a delta augmentation at T5 and T6 in the chronic stage.

Arrows indicate the statistically significant EEG amplitude alterations in the corresponding group (the subacute or chronic stage of the stroke vs. the control).

Fig. 3. Inter-hemispheric coherences following a minor stroke. The mean inter-hemispheric coherence spectra in the subacute $(n=10)$ and chronic $(n=9)$ stages of stroke patients vs. healthy controls $(\mathrm{n}=11)$.

In contrast to the parietal and temporal cortices $(\mathbf{C}, \mathbf{D})$ that expressed a generalized long-lasting desynchronization in all the frequency ranges ( $\mathrm{p} \leq 0.04$; lower panels), but with no change in 
777 alpha coherence ( $\mathrm{p} \geq 0.10)$, the frontal cortex exhibited an increased synchronization of slower

778 alpha $(\mathrm{p} \leq 0.02)$ in stroke patients vs. controls $(\mathbf{A}, \mathbf{B})$. This increased synchronization of slower

779 alpha was particularly expressed at F3-F4 electrodes (A), both in the subacute and the chronic 780 stage.

781 Arrows indicate the most consistent and significantly altered coherences in the corresponding 782 group (the subacute or chronic stage of stroke vs. the control).

783

Fig. 4. Intra-hemispheric coherences following a minor stroke. The mean intra-hemispheric 785 coherence spectra in the subacute $(n=10)$ and chronic $(n=9)$ stages of stroke patients vs. healthy controls $(\mathrm{n}=11)$.

787

788

789

790

791

792

793

794

795

796

797

798

799

In contrast to the increased synchronization of slower alpha in the ipsi-lesional hemisphere (D-F; $\mathrm{p} \leq 0.05)$ in the subacute and chronic stages of stroke patients vs. controls, the contra-lesional hemisphere exhibited a generalized but transient (only in the subacute stage) increase in theta synchronization $(\mathbf{A}-\mathbf{C} ; \mathrm{p} \leq 0.05)$ with no alteration to alpha synchronization.

Arrows indicate the most consistent and significantly altered coherences in the corresponding group (the subacute or chronic stage of stroke vs. the control).

Fig. 5. Topographic distribution of alpha PPs following a minor stroke. The group mean alpha PPs in the subacute $(n=10)$ and chronic $(n=9)$ stages of stroke patients vs. healthy controls $(n=11)$.

We have shown that the frontal cortex generally exhibits the greatest PP differences related to a slower alpha than all the other EEG channels in both the subacute (A) and chronic stages of stroke patients (B) vs. the controls, particularly at F4 (the red dashed circle). The persistence of 
800 stroke induced alpha PPs differences was additionally confirmed by the almost non-existing PP

801 differences between the subacute and chronic stages of the stroke patients (C). This result

802 indicates that the newly emerged slower alpha in stroke patients might be related to the

803 confirmed neuropathology of an ischemic stroke.

804 Thick circles: the mean PPs; thin circles: the mean PPs \pm SDs. Solid line: $0^{\circ}<$ PP $<180^{\circ}$;

805 dashed: $-180^{\circ}<\mathrm{PP}<0^{\circ}$. Referential circle with $\mathrm{PP}=60^{\circ}$, drawn in the lower right corner.

806 According to the angular nature of PP, the large solid circles (e.g. $\left.+175^{\circ}\right)$ have similar PP values

807 to the large dashed circles (e.g. $\left.-179^{\circ}\right)$.

808

809

Fig. 6. Topographic pattern of "alpha flow" following a minor stroke. A reconstruction of

810 "alpha flow" in healthy controls $(n=11)$, the subacute $(n=10)$ and the chronic $(n=9)$ stages of

811 stroke patients, based on the group mean alpha PPs distribution in the unit circle (A). The

812 topography of the descending order of PPs is plotted by arrows connecting the channel with a

813 higher PP to the channel with the next lower PP (B).

814 The greatest alterations in stroke patients vs. the controls were expressed as a permanently 815 established inter-hemispheric "alpha flow" (C).

816

817 Fig. 7. Correlations of MoCA and MoCA-MIS cognitive assessments and $\alpha A V G$ in the 818 subacute and chronic stages of stroke patients vs. the controls.

819 The statistically significant correlations of aAVG and MoCA (A-D) and MoCA-MIS (E-H) 820 scores in the subacute and chronic stages of stroke patients vs. the controls.

821 Bold numbers and asterisks indicate statistically significant correlations at $\mathrm{p} \leq 0.04$. 
Figure 1 (on next page)

Alterations in alpha frequency following a minor stroke

Group mean relative amplitude spectra of the frontal (A), parietal and temporal (B) EEG channels in the subacute $(n=10)$ and chronic $(n=9)$ stages of stroke patients vs. healthy controls $(n=11)$. We have demonstrated statistically significant slower alpha ( $\alpha A V G)$ at $F 3$, F4, F7, F8, P3 and P4 in the subacute stroke patients vs. the controls ( $p \leq 0.03)$. In the chronic stage, all stroke induced alpha frequency changes returned to the control values $(p \geq$ 0.06). Bold numbers and asterisks indicate a statistically significant decrease in $\alpha$ AVG following a stroke. 


\section{Figure 2 (on next page)}

EEG microstructure following minor stroke

Group mean probability density distributions (PDEs) of the delta, theta and beta relative amplitudes at the frontal (A), parietal and temporal (B) EEG channels in the subacute $(n=$ $10)$ and chronic $(n=9)$ stages of stroke patients vs. healthy controls $(n=11)$. The slower alpha in stroke patients with no amplitude change $(p \geq 0.14)$ was followed by a generally augmented theta ( $p \leq 0.03$ ), alongside an attenuated beta only within the frontal cortex and during both the subacute and chronic stage of the stroke $(p \leq 0.01)$. These stroke induced theta and beta amplitude alterations were followed by a delta augmentation at T5 and T6 in the chronic stage. Arrows indicate the statistically significant EEG amplitude alterations in the corresponding group (the subacute or chronic stage of the stroke vs. the control). 


\section{Figure 3 (on next page)}

Inter-hemispheric coherences following a minor stroke

The mean inter-hemispheric coherence spectra in the subacute $(n=10)$ and chronic $(n=9)$ stages of stroke patients vs. healthy controls $(n=11)$. In contrast to the parietal and temporal cortices (C, D) that expressed a generalized long lasting desynchronization in all the frequency ranges ( $p \leq 0.04$; lower panels), but with no change in alpha coherence $(p \geq$ $0.10)$, the frontal cortex exhibited an increased synchronization of slower alpha $(p \leq 0.02)$ in stroke patients vs. controls (A, B). This increased synchronization of slower alpha was particularly expressed at F3-F4 electrodes (A), both in the subacute and the chronic stage. Arrows indicate the most consistent and significantly altered coherences in the corresponding group (the subacute or chronic stage of stroke vs. the control). 


\section{Figure 4 (on next page)}

Intra-hemispheric coherences following a minor stroke

The mean intra-hemispheric coherence spectra in the subacute $(n=10)$ and chronic $(n=9)$ stages of stroke patients vs. healthy controls $(n=11)$. In contrast to the increased synchronization of slower alpha in the ipsi-lesional hemisphere (D-F; $p \leq 0.05)$ in the subacute and chronic stage of stroke patients vs. controls, the contra-lesional hemisphere exhibited a generalized but transient (only in the subacute stage) increase in theta synchronization (A-C; $\mathbf{P} \leq \mathbf{0 . 0 5}$ ) with no alteration to alpha synchronization. Arrows indicate the most consistent and significantly altered coherences in the corresponding group (the subacute or chronic stage of stroke vs. the control). 


\section{Figure $\mathbf{5}$ (on next page)}

Topographic distribution of alpha PPs following a minor stroke

The group mean alpha PPs in the subacute $(n=10)$ and chronic $(n=9)$ stages of stroke patients vs. healthy controls $(n=11)$. We have shown that the frontal cortex generally exhibits the greatest PP differences related to slower alpha than all the other EEG channels in both the subacute (A) and chronic stages of stroke patients (B) vs. the controls, particularly at F4 (the red dashed circle). The persistence of stroke induced alpha PPs differences was additionally confirmed by the almost non-existing PP differences between the subacute and chronic stages of the stroke patients $(\mathbf{C})$. This result indicates that the newly emerged slower alpha in stroke patients might be related to the confirmed neuropathology of an ischemic stroke. Thick circles: the mean PPs; thin circles: the mean PPs \pm SDs. Solid line: $0^{\circ}<$ PP $<$ $180^{\circ}$; dashed: $-180^{\circ}<\mathrm{PP}<0^{\circ}$. Referential circle with $\mathrm{PP}=60^{\circ}$, drawn in the lower right corner. According to the angular nature of PP, the large solid circles (e.g. $+175^{\circ}$ ) have similar PP values to the large dashed circles (e.g. $-179^{\circ}$ ). 

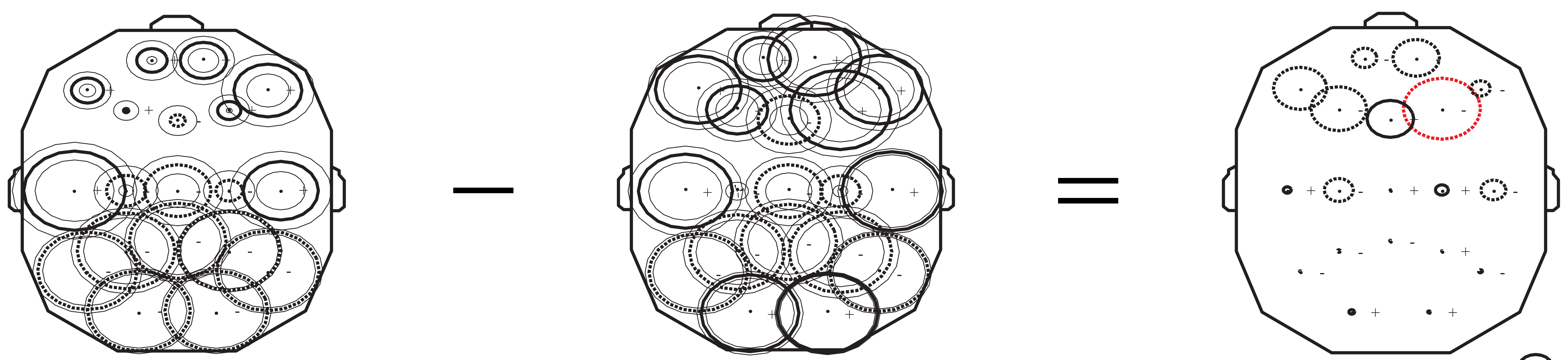

$60 \mathrm{deg}$

B

Chronic Stroke

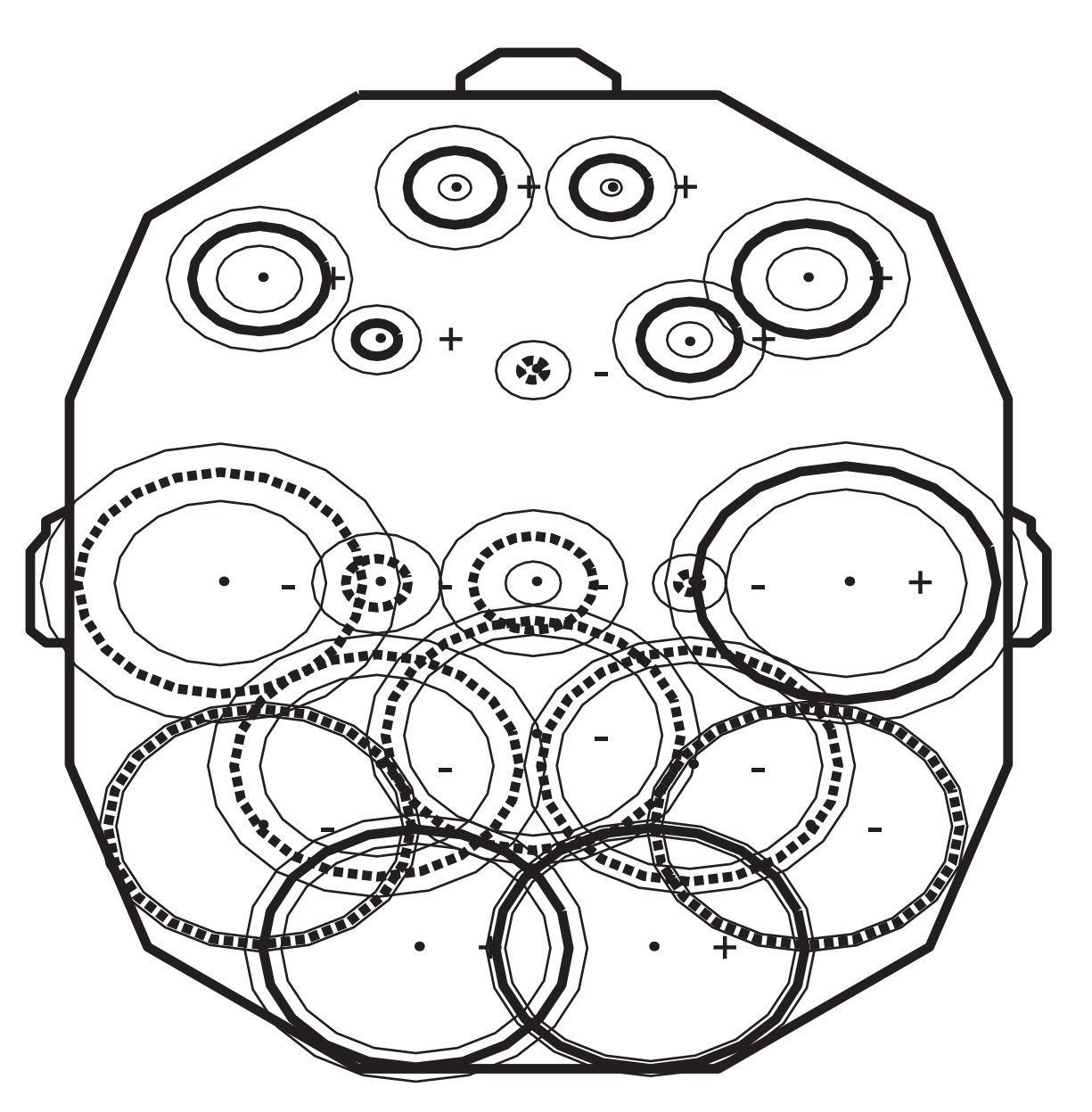

Control

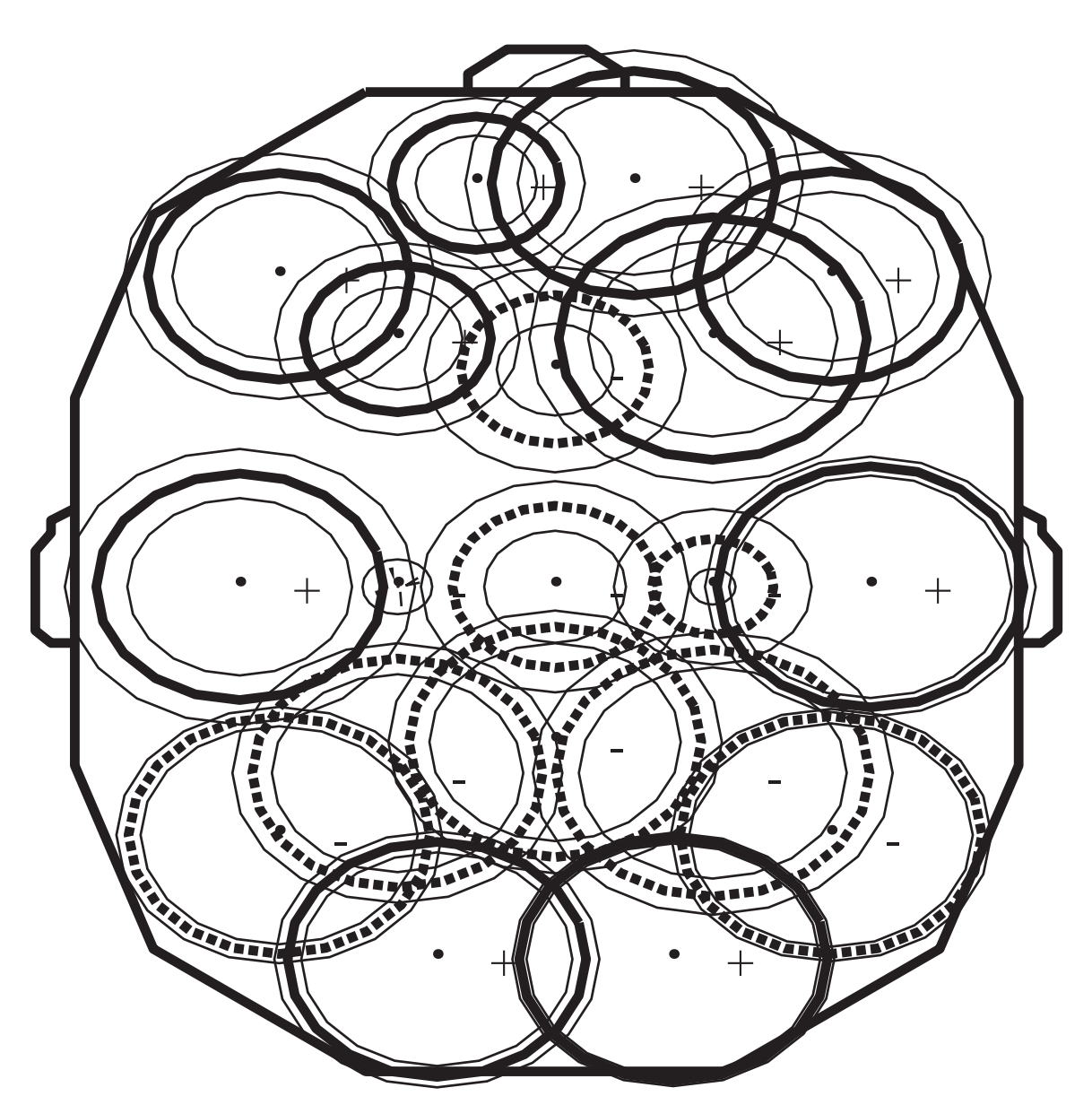

Chronic Stroke vs. Control

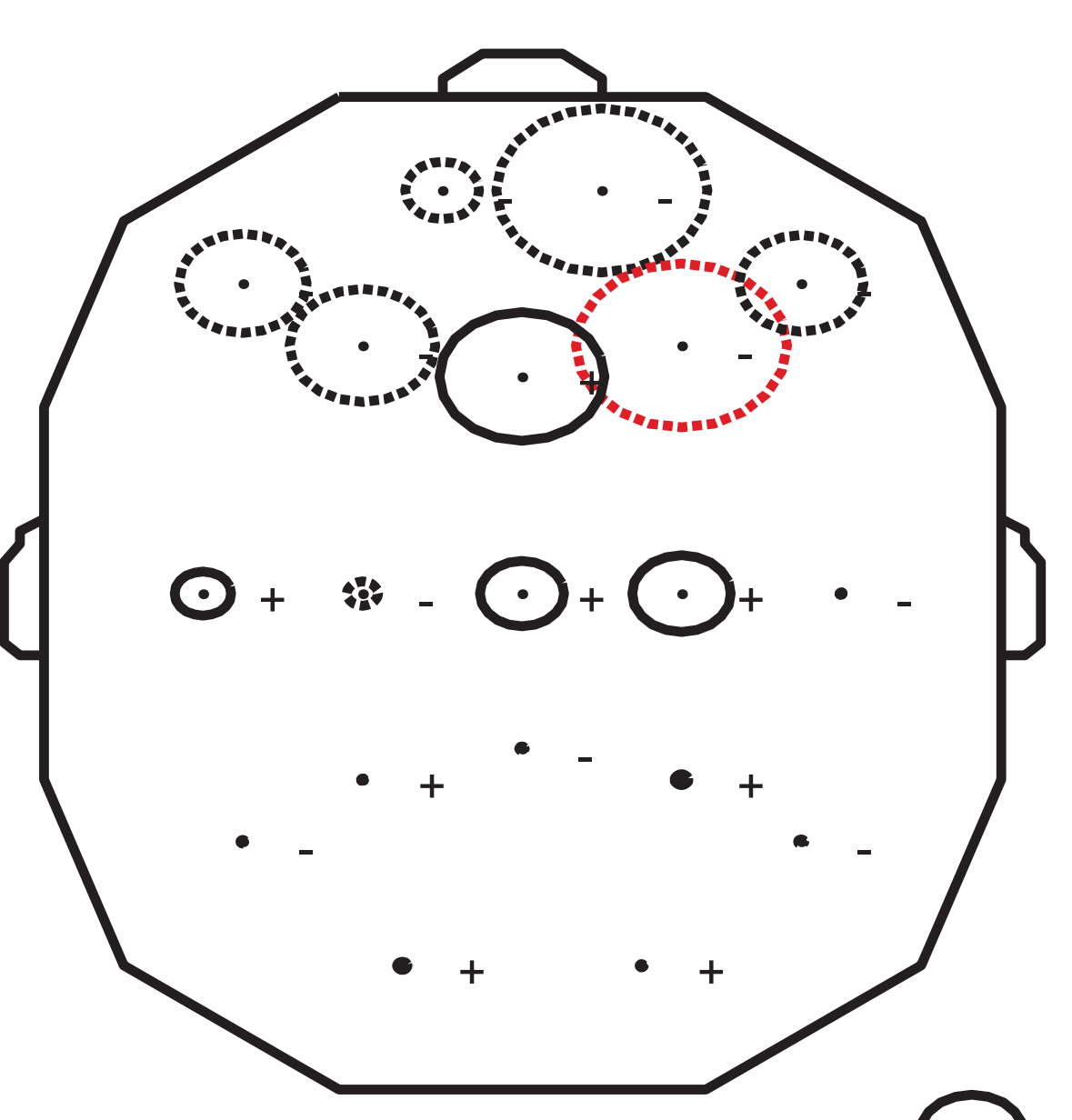

$60 \mathrm{deg}$
$\mathrm{C}$

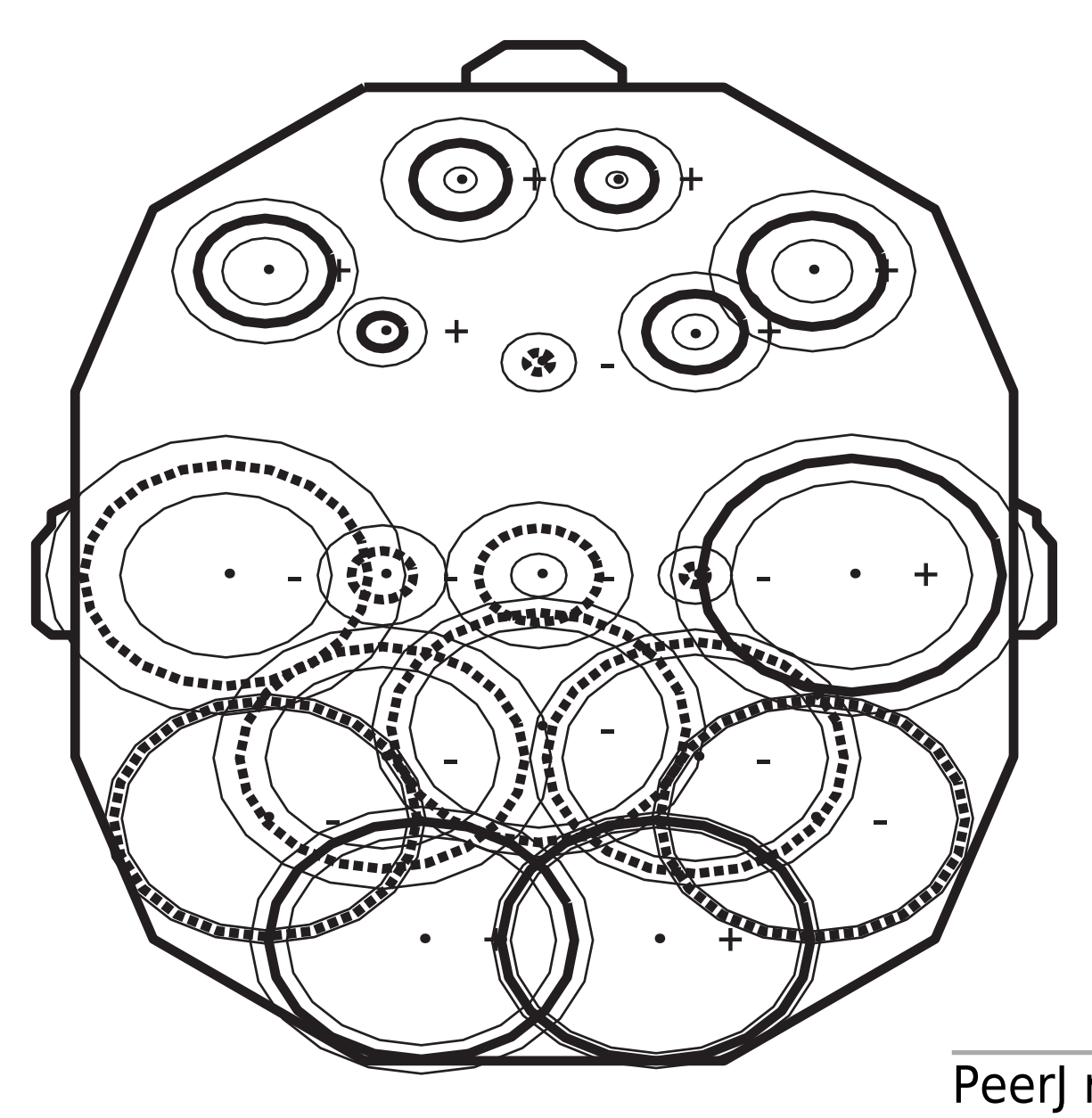

Subacute Stroke

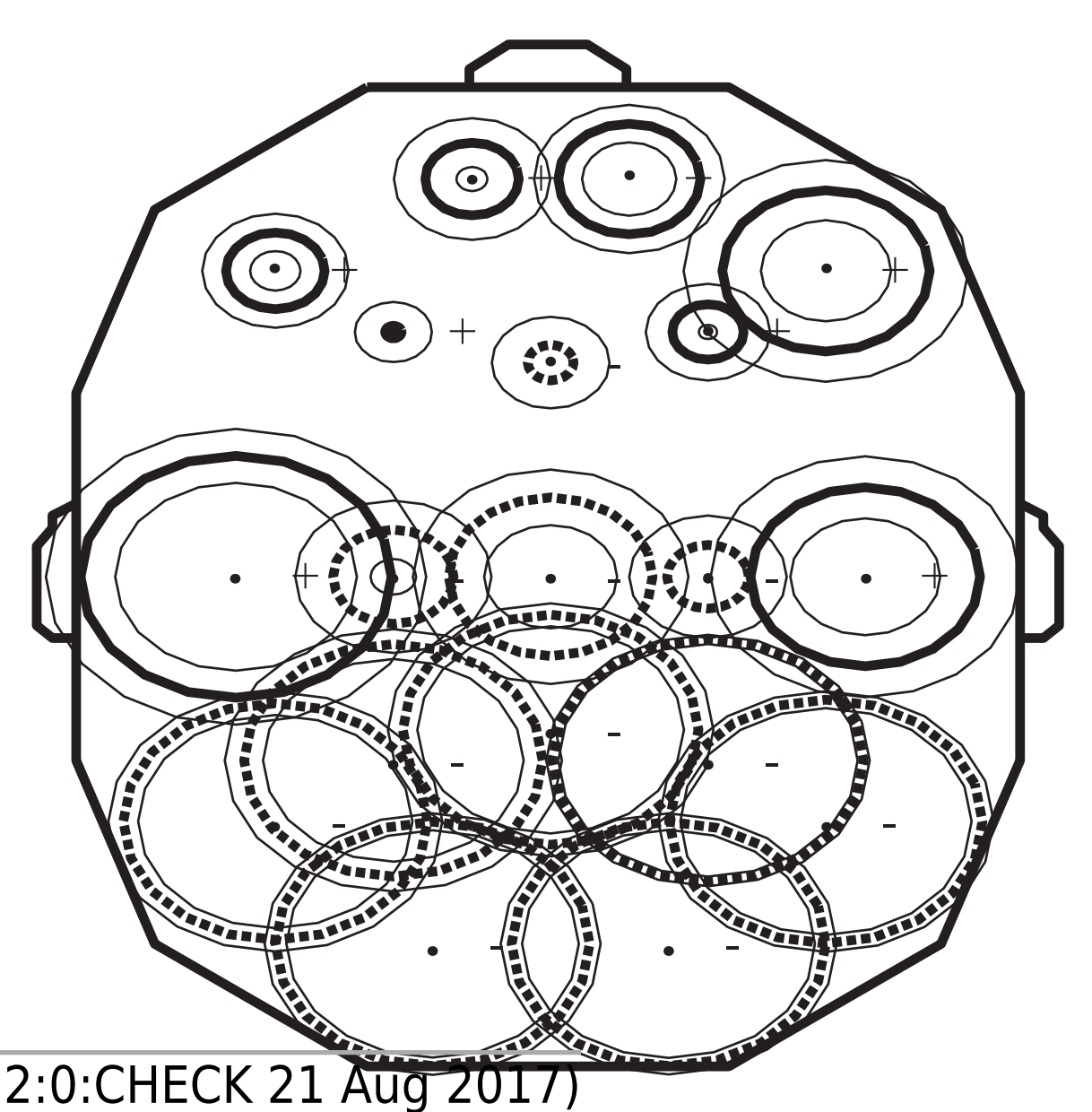

Chronic Stroke vs. Subacute Stroke

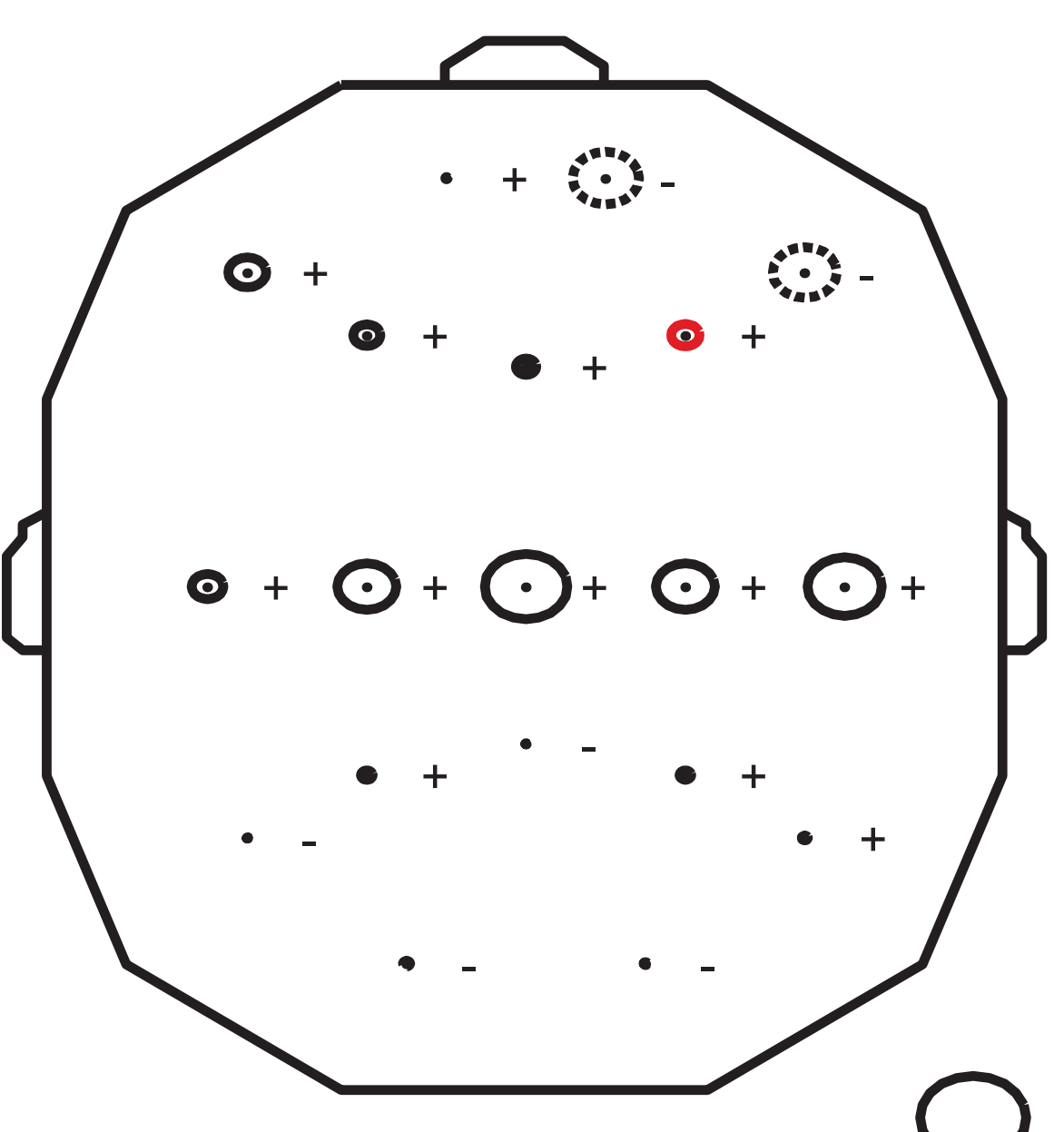


Figure $\mathbf{6}$ (on next page)

Topographic pattern of "alpha flow" following a minor stroke alpha

A reconstruction of "alpha flow" in healthy controls $(n=11)$, the subacute $(n=10)$ and the chronic $(n=9)$ stages of stroke patients, based on the group mean alpha PPs distribution on the unit circle (A). The topography of the descending order of PPs is plotted by arrows connecting the channel with a higher PP to the channel with the next lower PP (B). The greatest alterations in stroke patients vs. the controls were expressed as a permanently established inter-hemispheric "alpha flow" (C). 

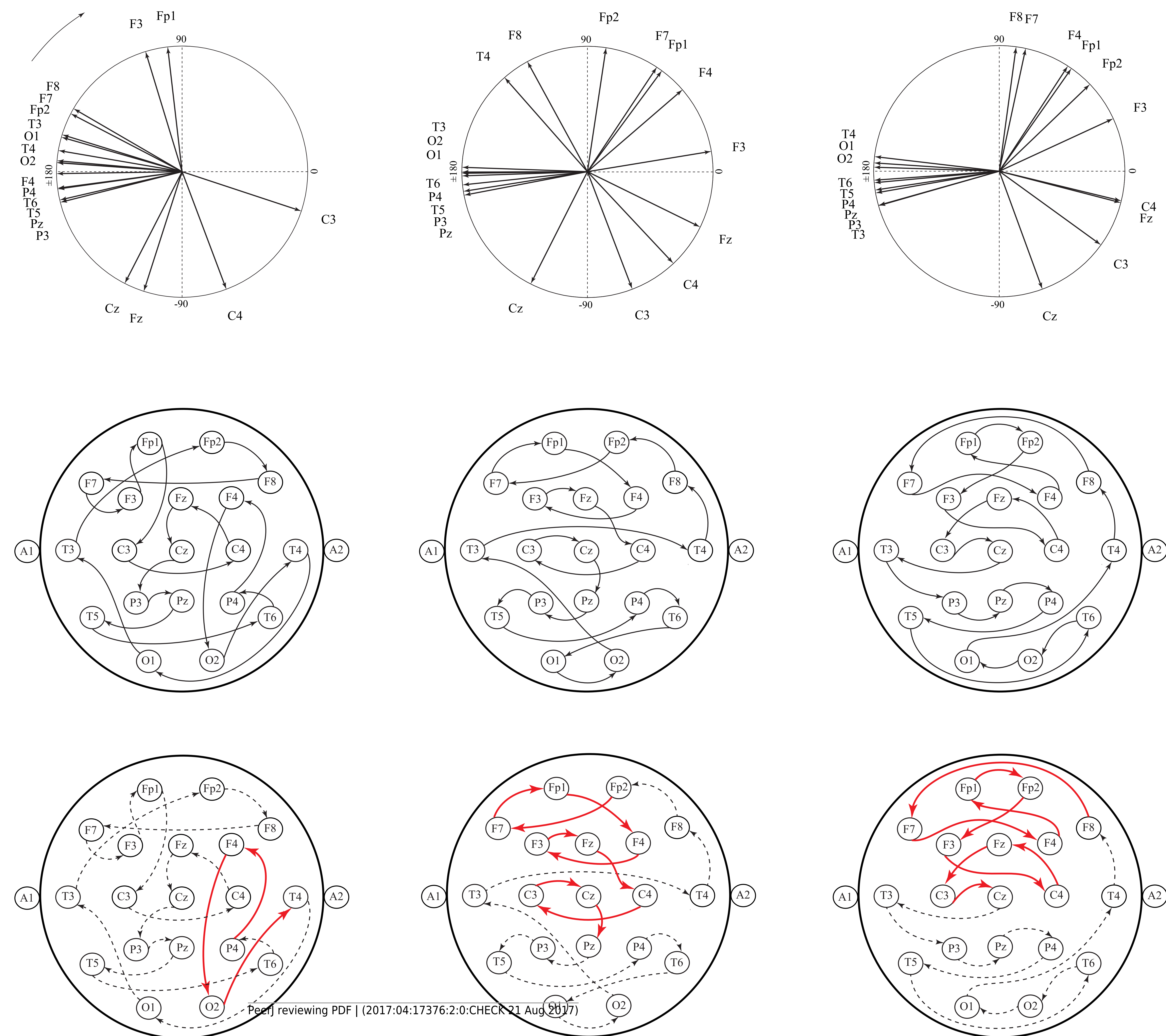
Figure 7 (on next page)

Correlations of MoCA and MoCA-MIS cognitive assessments and $\alpha$ AVG in subacute and chronic stages of stroke patients vs. the controls

The statistically significant correlations of $\alpha$ AVG and MoCA (A-D) and MoCA-MIS (E-H) scores in the subacute and chronic stages of stroke patients vs. the controls. Bold numbers and asterisks indicate statistically significant correlations at $p \leq 0.04$. 


\section{Table $\mathbf{1}$ (on next page)}

Demographic and clinical characteristics of stroke patients and healthy controls

Bold numbers indicate statistically significant values at $p \leq 0.05$. 
1 Table 1. Demographic and clinical characteristics of stroke patients and healthy controls.

\begin{tabular}{rlccc}
\hline & & Control & Subacute stroke & Chronic stroke \\
\cline { 2 - 4 } & Number & 11 & 10 & 9 \\
4 & Gender (m/f) & $5 / 6$ & $6 / 4$ & $5 / 4$ \\
5 & Age (years) & $60.64 \pm 9.91$ & $63.80 \pm 7.00$ & $63.89 \pm 6.83$ \\
6 & NIHSS (on admission) & $/$ & $8.20 \pm 3.05$ & $/$ \\
7 & NIHSS & $/$ & $2.50 \pm 0.85$ & $2.11 \pm 0.78$ \\
8 & Education (years) & $12.45 \pm 3.11$ & $9.80 \pm 2.70$ & $/$ \\
9 & MoCA score & $25.64 \pm 3.20$ & $\mathbf{2 0 . 8 0} \pm \mathbf{5 . 3 2}$ & $23.44 \pm 4.98$ \\
10 & MoCA-MIS & $11.91 \pm 2.98$ & $\mathbf{8 . 8 0} \pm \mathbf{3 . 5 2}$ & $10.22 \pm 3.35$ \\
\hline
\end{tabular}

11 Bold numbers indicate statistically significant values at $\mathrm{p} \leq 0.05$. 
Table 2 (on next page)

Patient's stroke details (Stroke neuropathology: the volume of lesions and the brain structures affected) 
1 Table 2. Patient's stroke details (Stroke neuropathology: the volume of lesions and the brain structures affected).

\begin{tabular}{|c|c|c|c|c|c|}
\hline $\begin{array}{c}\text { Patient } \\
\text { No. }\end{array}$ & $\begin{array}{c}\text { Gender } \\
(m / f)\end{array}$ & $\begin{array}{l}\text { Imaging } \\
\text { modality }\end{array}$ & $\begin{array}{c}\text { Volume } \\
\left(\mathrm{cm}^{3}\right)\end{array}$ & Subcortical structures affected & Cortical structres affected \\
\hline 1 & $\mathrm{~m}$ & $\mathrm{CT}$ & 18.90 & putamen & insular cortex \\
\hline 2 & $\mathrm{~m}$ & $\mathrm{CT}$ & 9.11 & putamen & \\
\hline 3 & $\mathrm{~m}$ & $\mathrm{CT}$ & 0.90 & & insular cortex \\
\hline 4 & $\mathrm{f}$ & CT & 13.19 & putamen & \\
\hline 5 & $\mathrm{~m}$ & $\mathrm{CT}$ & 9.04 & & superior temporal gyrus \\
\hline 6 & $\mathrm{f}$ & $\mathrm{CT}$ & 14.04 & putamen & insular cortex, superior temporal gyrus \\
\hline 7 & $\mathrm{f}$ & $\mathrm{CT}$ & 0.96 & putamen & \\
\hline 8 & $\mathrm{~m}$ & MRI & 10.20 & putamen, globus pallidus & \\
\hline 9 & $\mathrm{~m}$ & $\mathrm{CT}$ & 15.84 & & transverse temporal gyrus, supramarginal gyrus \\
\hline 10 & $\mathrm{f}$ & MRI & 1.20 & & precentral gyrus, postcentral gyrus \\
\hline
\end{tabular}

2 


\section{Table 3(on next page)}

Correlations of MoCA and MoCA-MIS cognitive assessments and $\alpha$ AVG in the subacute and chronic stages of stroke patients vs. healthy controls

Bold numbers indicate statistically significant correlations at $p \leq 0.05$. 

patients vs. healty controls.

\begin{tabular}{|c|c|c|c|c|c|c|c|c|c|}
\hline & & \multicolumn{8}{|c|}{ MoCA vs. $\alpha A V G$} \\
\hline & & F3 & F4 & F7 & F8 & P3 & $\mathbf{P 4}$ & T5 & T6 \\
\hline \multirow{2}{*}{ Control } & rho & 0.06 & -0.02 & 0.13 & 0.10 & 0.01 & -0.20 & -0.23 & -0.24 \\
\hline & $\mathrm{p}$ & 0.87 & 0.95 & 0.70 & 0.78 & 0.97 & 0.56 & 0.50 & 0.47 \\
\hline \multirow{2}{*}{ Subacute Stroke } & rho & 0.51 & 0.74 & 0.09 & 0.52 & 0.65 & 0.77 & 0.53 & 0.74 \\
\hline & $\mathrm{p}$ & 0.13 & 0.02 & 0.82 & 0.12 & 0.04 & 0.01 & 0.12 & $\mathbf{0 . 0 2}$ \\
\hline \multirow{4}{*}{ Chronic Stroke } & rho & -0.04 & 0.34 & 0.08 & 0.39 & 0.13 & 0.15 & 0.22 & 0.19 \\
\hline & $\mathrm{p}$ & 0.92 & 0.38 & 0.83 & 0.30 & 0.75 & 0.70 & 0.57 & 0.63 \\
\hline & & \multicolumn{8}{|c|}{ MoCA-MIS vs. $\alpha A V G$} \\
\hline & & F3 & F4 & F7 & F8 & $\mathbf{P 3}$ & $\mathbf{P 4}$ & T5 & T6 \\
\hline \multirow{2}{*}{ Control } & rho & 0.23 & 0.14 & 0.30 & 0.21 & 0.20 & 0.00 & -0.02 & -0.04 \\
\hline & $\mathrm{p}$ & 0.49 & 0.69 & 0.37 & 0.54 & 0.57 & 1.00 & 0.95 & 0.92 \\
\hline \multirow{2}{*}{ Subacute Stroke } & rho & 0.28 & 0.28 & 0.37 & 0.16 & 0.40 & 0.13 & 0.57 & 0.24 \\
\hline & $\mathrm{p}$ & 0.44 & 0.43 & 0.30 & 0.66 & 0.25 & 0.71 & 0.09 & 0.51 \\
\hline \multirow{2}{*}{ Chronic Stroke } & rho & 0.56 & 0.63 & 0.61 & 0.52 & 0.72 & 0.92 & 0.77 & 0.92 \\
\hline & $\mathrm{p}$ & 0.12 & 0.07 & 0.08 & 0.15 & $\mathbf{0 . 0 3}$ & $<0.01$ & 0.02 & $<0.01$ \\
\hline
\end{tabular}

3

4 Bold numbers indicate statistically significant correlations at $\mathrm{p} \leq 0.05$ 